\title{
RANDOM ATTRACTORS FOR STOCHASTIC EVOLUTION EQUATIONS DRIVEN BY FRACTIONAL BROWNIAN MOTION*
}

\author{
HONGJUN GAO ${ }^{\dagger}$, MARÍA J. GARRIDO-ATIENZA ${ }^{\ddagger}$, AND BJÖRN SCHMALFUSS ${ }^{\S}$
}

\begin{abstract}
The main goal of this article is to prove the existence of a random attractor for a stochastic evolution equation driven by a fractional Brownian motion with Hurst parameter $H \in$ $(1 / 2,1)$. We would like to emphasize that we do not use the usual cohomology method, consisting of transforming the stochastic equation into a random one, but we deal directly with the stochastic equation. In particular, in order to get adequate a priori estimates of the solution needed for the existence of an absorbing ball, we will introduce stopping times to control the size of the noise. In the first part of this article we shall obtain the existence of a pullback attractor for the nonautonomous dynamical system generated by the pathwise mild solution of an nonlinear infinitedimensional evolution equation with a nontrivial Hölder continuous driving function. In the second part, we shall consider the random setup: stochastic equations having as a driving process a fractional Brownian motion with $H \in(1 / 2,1)$. Under a smallness condition for that noise we will show the existence and uniqueness of a random attractor for the stochastic evolution equation.
\end{abstract}

Key words. fractional derivatives, pathwise mild solutions, nonautonomous and random dynamical systems, fractional Brownian motion, pullback and random attractors

AMS subject classifications. 37L55, 60H15, 37L25, 35R60, 58B99

DOI. $10.1137 / 130930662$

1. Introduction. This article shows the existence of random attractors for a new class of stochastic evolution equations. These equations contain a nontrivial fractional noise with a Hurst parameter $H>1 / 2$. In particular, it generalizes the results of the conference proceedings by Garrido-Atienza, Maslowski, and Schmalfuss [12], where random attractors are studied for ordinary stochastic equations containing a fractional noise. The idea of this article is based on the modern theory of stochastic integration for fractional Brownian motions (fBm) with $H>1 / 2$; see, for instance, Zähle [27]. These integrals are related to the Young integration [26] and one of its presentations is based on a sort of generalized integration by parts formula with respect to fractional derivatives. The main advantage of this integration with respect to the classical Itô integration theory, where the integrator is a white noise (or equivalently a fractional Brownian motion with Hurst parameter $H=1 / 2$ ), is that integrals can be defined pathwise. However, Itô integrals are only defined almost surely and exceptional sets may depend on the integrand. But this fact contradicts the definition of a random dynamical system, where initial state dependent exceptional sets are not allowed. By

\footnotetext{
* Received by the editors July 25, 2013; accepted for publication (in revised form) April 4, 2014; published electronically July 1, 2014.

http://www.siam.org/journals/sima/46-4/93066.html

$\dagger$ Institute of Mathematics, School of Mathematical Sciences, Nanjing Normal University, Nanjing 210046, China (gaohj@njnu.edu.cn). This author was supported by China NSF grant 11171158, National Basic Research Program of China (973 Program) 2013CB834100, the Natural Science Foundation of Jiangsu Province (BK2011777), Qing Lan and "333" Project of Jiangsu Province, and the NSF of the Jiangsu Higher Education Committee of China (11KJA110001) and the Jiangsu Collaborative Innovation Center for Climate Change.

${ }^{\ddagger}$ Dpto. Ecuaciones Diferenciales y Análisis Numérico, Universidad de Sevilla, 41080 Sevilla, Spain (mgarrido@us.es). This author was partially supported by the European Funds for Regional Development and Ministerio de Economia y Competitividad (Spain) under grant MTM2011-22411.

§Institut für Mathematik, 07737 Jena, Germany (bjoern.schmalfuss@uni-jena.de). This author was partially supported by the European Funds for Regional Development and Ministerio de Economia y Competitividad (Spain) under grant MTM2011-22411.
} 
using fractional derivatives to define stochastic integrals we are able to avoid this dependence on exceptional sets.

We remark that the results presented in this article do not cover the white noise case. For the existence of pathwise solutions for this case (and more generally for the cases in which $H \in(1 / 3,1 / 2])$ we refer to [10], [11] and the forthcoming paper [9]. We want to emphasize here that the techniques to obtain such a pathwise solution are qualitatively different from the methods presented in this article.

This article is divided into two parts. In the first part, sections 2 and 3, we mention that nonlinear infinite-dimensional evolution equations driven by a Hölder continuous function with Hölder index greater than $1 / 2$ have a pathwise mild solution which generates a nonautonomous dynamical system, and we obtain the existence of a unique pullback attractor associated to it if we restrict this dynamical system to a discrete time set. Existence results for this kind of equation have been studied in Maslowski and Nualart [20] and in Garrido-Atienza, Lu, and Schmalfuss [8] when the driving function is an $\mathrm{fBm}$ with $H>1 / 2$, and very recently by Chen, Gao, Garrido-Atienza, and Schmalfuss [5]. In this last paper, the authors were able to overcome the lack of the regularity of the semigroup generated by the linear part of the evolution equation by considering suitable modifications of the space of $\beta$-Hölder continuous functions as phase space, where $\beta>1 / 2$ is related to the Hölder index of the driving function, and they established the existence and uniqueness of a pathwise mild solution. These results are thus briefly included as a preliminary step, where the construction of the integral for Hölder continuous integrators is the main tool. Then, by standard arguments we can consider the pathwise mild solution to be a nonautonomous dynamical system.

According to Flandoli and Schmalfuss [7] or Schmalfuss [23], in order to prove the existence of a nonautonomous or pullback attractor, appropriate a priori estimates of the solution are necessary. In particular (see Theorem 2.2 below), we should construct a compact pullback absorbing set belonging to a certain family. However, by the particular structure of the estimates of the pathwise integrals, the standard Gronwall lemma is not available and, therefore, we have to formulate a special Gronwall lemma for discrete time, which will be shown to work only when the estimates of these integrals are not too large. To ensure that this smallness condition holds true we will introduce (non-Markovian) stopping times with the property that they themselves satisfy the cocycle property, and assuming that these stopping times have special asymptotic properties, the existence of a unique pullback attractor is obtained.

Then, in the second part of the article (section 4), we show how to adapt the previous results to obtain the random attractor associated to stochastic evolution equations driven by an $\mathrm{fBm}$ with $H>1 / 2$. In fact, by choosing the canonical version of the $\mathrm{fBm}$, we show that the nonautonomous dynamical system becomes a random dynamical system and that the stopping times are measurable. In addition, it suffices to choose fractional noises with covariance operators having small trace, since this condition ensures that the required asymptotical properties of the stopping times hold with probability one. All the previous study finally concludes with the existence and uniqueness of a random attractor.

To finish the introduction, we would like to stress that there are a few papers whose main concern is the study of the random attractor for stochastic differential equations and stochastic partial differential equations (SPDEs) driven by an fBm with $H>1 / 2$; see [13], [15], [16], and [17]. But in all these papers the noise has a special structure (more precisely, either pure additive noise or a very simple multiplicative noise) making it possible to use the cohomology method, and hence the random attractor is obtained by transforming the stochastic equation into a random one. Our 
aim here is to consider more general noisy terms with the challenge of getting the random attractor with no transformation. We also should mention that in [12] the existence of the random attractor for a stochastic differential equation driven by such an $\mathrm{fBm}$ was successfully obtained without using the cohomology method. Indeed the technique we are going to follow here has some similarities to the one used in [12], in the sense that there the authors also considered stopping times and the discrete nonautonomous dynamical system as a preliminary step to get the random attractor. However, since the nature of infinite dynamical systems is different from that of their finite counterparts, the construction of the absorbing set in this paper in much more involved, mainly due to the lack of regularity of the semigroup and the way of getting the compactness of the absorbing ball.

2. Preliminaries. In this section we collect definitions and properties of dynamical systems and present the construction of the integral with Hölder continuous integrator and Hölder exponent greater than 1/2. Although this construction has already been done in the recent paper [5], we present it here for the sake of completeness, since a good understanding of this integral is the starting point to obtain the cocycle property and afterward the random attractor associated to the corresponding stochastic evolution equation.

2.1. Dynamical systems. Let $(V,|\cdot|)$ be a Banach space and let $T^{+}=R^{+}$or $Z^{+}$. A mapping $\varphi: T^{+} \times V \rightarrow V$ having the semigroup property

$$
\varphi(t, \cdot) \circ \varphi\left(\tau, u_{0}\right)=\varphi\left(t+\tau, u_{0}\right), \quad \varphi\left(0, u_{0}\right)=u_{0} \quad t, \tau \in T^{+}, \quad u_{0} \in V,
$$

is called an (autonomous) dynamical system. A dynamical system $\varphi$ has a global attractor $\mathcal{A} \subset V$ with respect to the set system $\mathcal{D}$, consisting of the bounded sets of $V$, if $\mathcal{A}$ is nonempty, compact, and invariant, that is,

$$
\varphi(t, \mathcal{A})=\mathcal{A} \quad \text { for all } t \in T^{+}
$$

and

$$
\lim _{T^{+} \ni t \rightarrow \infty} \operatorname{dist}(\varphi(t, D), \mathcal{A})=0 \quad \text { for every } D \in \mathcal{D} .
$$

$\operatorname{dist}(X, Y)$ denotes the Hausdorff semidistance defined by

$$
\operatorname{dist}(X, Y)=\sup _{x \in X} \inf _{y \in Y}|x-y| .
$$

A comprehensive presentation of the concept of attractors can be found in the work by Babin and Vishik [2], Hale [18], or Temam [25].

We want to consider a generalization of the concept of global attractors to nonautonomous and random dynamical systems given by the pullback attractors. As a first ingredient, for the time set $T=R$ or $Z$, we introduce the flow $\left(\theta_{t}\right)_{t \in T}$ on the set $\Omega$ of nonautonomous perturbations by

$$
\begin{aligned}
& \theta: T \times \Omega \rightarrow \Omega, \\
& \theta_{t} \circ \theta_{\tau}=\theta_{t+\tau}, \quad \theta_{0} \omega=\omega \quad \text { for } t, \tau \in T, \omega \in \Omega .
\end{aligned}
$$

The easiest example for such a flow is given by $\Omega=T$ and $\theta_{j} i=i+j$ for $j \in T$. 
As a generalization of the semigroup property we consider a cocycle to be a mapping $\varphi: T^{+} \times \Omega \times V \rightarrow V$ such that

$$
\begin{aligned}
\varphi\left(t+\tau, \omega, u_{0}\right) & =\varphi\left(t, \theta_{\tau} \omega, \cdot\right) \circ \varphi\left(\tau, \omega, u_{0}\right), \\
\varphi\left(0, \omega, u_{0}\right) & =u_{0}
\end{aligned}
$$

for all $t, \tau \in T^{+}, u_{0} \in V$, and $\omega \in \Omega$. $\varphi$ is also called a nonautonomous dynamical system.

Let us now equip $(\Omega, \theta)$ with a measurable structure. Consider the probability space $(\Omega, \mathcal{F}, P)$ where $\mathcal{F}$ is a $\sigma$-algebra on $\Omega$ and $P$ is a measure, which is supposed to be invariant and ergodic with respect to $\theta$. Then $(\Omega, \mathcal{F}, P, \theta)$ is called an ergodic metric dynamical system. A $\mathcal{B}\left(T^{+}\right) \otimes \mathcal{F} \otimes \mathcal{B}(V), \mathcal{B}(V)$ measurable mapping $\varphi$ having the cocycle property $(2.1)$ is called a random dynamical system with respect to the metric dynamical system $(\Omega, \mathcal{F}, P, \theta)$.

Before we give the notion of an attractor for a nonautonomous dynamical system we introduce set systems that will be attracted by that attractor. Let $\mathcal{D}$ be a set of families $(D(\omega))_{\omega \in \Omega}$ such that $\emptyset \neq D(\omega) \subset V$ and satisfies a general property $\mathcal{P}$, which will be determined later. In addition we assume the following completeness condition for $\mathcal{D}$ : let $D^{\prime}=\left(D^{\prime}(\omega)\right)_{\omega \in \Omega}$ such that $\emptyset \neq D^{\prime}(\omega) \subset V$ and satisfies the property $\mathcal{P}$; if there exists a $D \in \mathcal{D}$ such that $D^{\prime}(\omega) \subset D(\omega)$ for $\omega \in \Omega$, then $D^{\prime} \in \mathcal{D}$. For a given $\nu>0$, an example of such a system is the backward $\nu$-exponentially growing sets $\mathcal{D}_{V}^{\nu}: D=(D(\omega))_{\omega \in \Omega}$ is an element of this set system if there exists a mapping $\Omega \ni \omega \mapsto r(\omega) \in R^{+}$such that $\emptyset \neq D(\omega) \subset B_{V}(0, r(\omega))$ and

$$
\limsup _{T \ni t \rightarrow-\infty} \frac{\log ^{+} r\left(\theta_{t} \omega\right)}{|t|}<\nu
$$

$B_{V}(0, r(\omega))$ denotes the ball in $V$ with center 0 and radius $r(\omega)$. We also stress that sometimes we write $\mathcal{D}_{Z, V}^{\nu}$ or $\mathcal{D}_{R, V}^{\nu}$ to emphasize the corresponding time set. We note that it is enough to consider these properties along an orbit $\bigcup_{t \in T}\left\{\theta_{t} \omega\right\}$. However, we define families for $(D(\omega))_{\omega \in \Omega}$.

We now consider these families of sets in a random setup. Assuming that $V$ is a separable Banach space, we consider $D=(D(\omega))_{\omega \in \Omega}$ such that $\emptyset \neq D(\omega) \subset V$ is closed and verifies the following property $\mathcal{P}$ : the mapping

$$
\omega \mapsto \operatorname{dist}(\{u\}, D(\omega))
$$

is a random variable for $u \in V$. Then $D$ is a random set; see Castaing and Valadier [4, Chapter III]. Indeed, we are interested in considering the set $\hat{\mathcal{D}}$ of random sets having backward and forward subexponential growth $(\nu=0)$, which means that for a random set $D$ there exists a positive random variable $r$ such that $D(\omega) \subset B_{V}(0, r(\omega))$ and

$$
\lim _{T \ni t \rightarrow \pm \infty} \frac{\log ^{+} r\left(\theta_{t} \omega\right)}{|t|}=0 \quad \text { for } \omega \in \Omega .
$$

These sets are also called the random tempered sets. In this case we are only interested in the time set $T=R$.

By the following lemma it is only interesting to consider the case $\nu=0$.

Lemma 2.1. Let $(\Omega, \mathcal{F}, P)$ be an ergodic metric dynamical system. If $D$ is a $\nu$ exponentially growing random set $(\nu<\infty)$, then $D$ is just subexponentially growing. 
The result follows directly, since by Arnold [1, Proposition 4.1.3] we have that

$$
\lim _{t \rightarrow+\infty} \frac{\log ^{+} r\left(\theta_{t} \omega\right)}{t}=\lim _{t \rightarrow-\infty} \frac{\log ^{+} r\left(\theta_{t} \omega\right)}{|t|} \in\{0,+\infty\} .
$$

A family $\mathcal{A}=(\mathcal{A}(\omega))_{\omega \in \Omega}$ is a pullback attractor for the nonautonomous dynamical system $\varphi$ with respect to $\mathcal{D}$ if $\mathcal{A} \in \mathcal{D}$ is nonempty, compact, and invariant in the sense that

$$
\varphi(t, \omega, \mathcal{A}(\omega))=\mathcal{A}\left(\theta_{t} \omega\right) \quad \text { for all } t \in T^{+}, \omega \in \Omega,
$$

and

$$
\lim _{T \ni t \rightarrow+\infty} \operatorname{dist}\left(\varphi\left(t, \theta_{-t} \omega, D\left(\theta_{-t} \omega\right)\right), \mathcal{A}(\omega)\right)=0 \quad \text { for all } \omega \in \Omega, D \in \mathcal{D} .
$$

In the case that $\varphi$ is a random dynamical system and $\hat{\mathcal{D}}$ consists of the random tempered sets introduced above, we call $\mathcal{A}$ a random attractor. This notion has been introduced in Schmalfuss [23].

Finally, a family $B=(B(\omega))_{\omega \in \Omega}$ is called pullback absorbing for $\mathcal{D}$ if

$$
\varphi\left(t, \theta_{-t} \omega, D\left(\theta_{-t} \omega\right)\right) \subset B(\omega) \text { for } t \geq \tilde{T}(D, \omega) \in T^{+}
$$

for any $D \in \mathcal{D}$ and $\omega \in \Omega$. $\tilde{T}(D, \omega)$ is the so-called absorption time.

We have the following main theorem about the existence of a pullback/random attractor (see Flandoli and Schmalfuss [7] or Schmalfuss [24]).

TheOREM 2.2. Let $\varphi$ be a continuous nonautonomous dynamical system. Suppose that $\varphi$ has a compact pullback absorbing set $B$ in $\mathcal{D}$. Then $\varphi$ has a unique pullback attractor with respect to $\mathcal{D}$. If $\varphi$ is a random dynamical system and $\mathcal{D}=\hat{\mathcal{D}}$ consists of the tempered sets, then the associated pullback attractor is a random attractor. In both cases

$$
\mathcal{A}(\omega)=\bigcap_{\tau \geq T_{0}(B, \omega)} \overline{\bigcup_{t \geq \tau} \varphi\left(t, \theta_{-t} \omega, B\left(\theta_{-t} \omega\right)\right)}
$$

where $T_{0}(B, \omega)$ is the absorption time corresponding to $B$.

2.2. Integrals in Hilbert spaces for Hölder continuous integrators with Hölder exponent $>\mathbf{1 / 2}$. We begin this subsection by introducing some function spaces. Assume $(V,(\cdot, \cdot),|\cdot|)$ is a separable Hilbert space with the complete orthonormal base $\left(e_{i}\right)_{i \in N}$. Let $C^{\beta}\left(\left[T_{1}, T_{2}\right] ; V\right)$ be the Banach space of Hölder continuous functions with exponent $\beta>0$ having values in $V$. A norm on this space is given by

$$
\|u\|_{\beta}=\|u\|_{\beta, T_{1}, T_{2}}=\sup _{s \in\left[T_{1}, T_{2}\right]}|u(s)|+\left|\|u \mid\|_{\beta, T_{1}, T_{2}}\right.
$$

with

$$
|||u| \|_{\beta, T_{1}, T_{2}}=\sup _{T_{1} \leq s<t \leq T_{2}} \frac{|u(t)-u(s)|}{|t-s|^{\beta}} .
$$

$C\left(\left[T_{1}, T_{2}\right] ; V\right)$ denotes the space of continuous functions on $\left[T_{1}, T_{2}\right]$ with values in $V$ with finite supremum norm, and let $C^{\beta, \sim}\left(\left[T_{1}, T_{2}\right] ; V\right) \subset C\left(\left[T_{1}, T_{2}\right] ; V\right)$ be equipped with the norm

$$
\|u\|_{\beta, \sim}=\|u\|_{\beta, \sim, T_{1}, T_{2}}=\sup _{s \in\left[T_{1}, T_{2}\right]}|u(s)|+\sup _{T_{1}<s<t \leq T_{2}}\left(s-T_{1}\right)^{\beta} \frac{|u(t)-u(s)|}{|t-s|^{\beta}} .
$$

Copyright (c) by SIAM. Unauthorized reproduction of this article is prohibited. 
For every $\rho>0$ we can consider the equivalent norm

$$
\begin{aligned}
\|u\|_{\beta, \rho, \sim, T_{1}, T_{2}}= & \sup _{s \in\left[T_{1}, T_{2}\right]} e^{-\rho\left(s-T_{1}\right)}|u(s)| \\
& +\sup _{T_{1}<s<t \leq T_{2}}\left(s-T_{1}\right)^{\beta} e^{-\rho\left(t-T_{1}\right)} \frac{|u(t)-u(s)|}{|t-s|^{\beta}} .
\end{aligned}
$$

When no confusion is possible, we will write $\|u\|_{\beta, \rho, \sim}$ instead of $\|u\|_{\beta, \rho, \sim, T_{1}, T_{2}}$.

Lemma 2.3 (see $[5]) . C^{\beta, \sim}\left(\left[T_{1}, T_{2}\right] ; V\right)$ is a Banach space.

Now we introduce integrals where the integrator is a Hölder continuous function. The definition of these integrals shall have as a foundation the definition and properties of the pathwise integrals given by Zähle [27]. Let us then assume that $\tilde{V}, \hat{V}$ are separable Hilbert spaces; then for $0<\alpha<1$ and general measurable functions $K$ : $\left[T_{1}, T_{2}\right] \mapsto \hat{V}$ and $\omega:\left[T_{1}, T_{2}\right] \mapsto \tilde{V}$, we define the following Weyl fractional derivatives:

$$
\begin{aligned}
D_{T_{1}+}^{\alpha} K[r] & =\frac{1}{\Gamma(1-\alpha)}\left(\frac{K(r)}{\left(r-T_{1}\right)^{\alpha}}+\alpha \int_{T_{1}}^{r} \frac{K(r)-K(q)}{(r-q)^{1+\alpha}} d q\right) \in \hat{V}, \\
D_{T_{2}-}^{1-\alpha} \omega_{T_{2}-}[r] & =\frac{(-1)^{1-\alpha}}{\Gamma(\alpha)}\left(\frac{\omega(r)-\omega\left(T_{2}-\right)}{\left(T_{2}-r\right)^{1-\alpha}}+(1-\alpha) \int_{r}^{T_{2}} \frac{\omega(r)-\omega(q)}{(q-r)^{2-\alpha}} d q\right) \in \tilde{V},
\end{aligned}
$$

where $\omega_{T_{2}-}(r)=\omega(r)-\omega\left(T_{2}-\right), \omega\left(T_{2}-\right)$ is the left side limit of $\omega$ at $T_{2}$, and $\Gamma(\cdot)$ is the Gamma function.

Let $f \in L_{1}\left(\left(T_{1}, T_{2}\right) ; R\right)$ and $\alpha>0$. Following Samko, Kilbas, and Marichev [22], the left-sided and right-sided fractional Riemann-Liouville integrals of $f$ of order $\alpha$ are defined for almost all $x \in\left(T_{1}, T_{2}\right)$ by

$$
\begin{aligned}
& I_{T_{1}+}^{\alpha} f(x)=\frac{1}{\Gamma(\alpha)} \int_{T_{1}}^{x}(x-r)^{\alpha-1} f(r) d r, \\
& I_{T_{2}-}^{\alpha} f(x)=\frac{(-1)^{-\alpha}}{\Gamma(\alpha)} \int_{x}^{T_{2}}(r-x)^{\alpha-1} f(r) d r,
\end{aligned}
$$

respectively. Denote by $I_{T_{1}+}^{\alpha}\left(L_{p}\left(\left(T_{1}, T_{2}\right) ; R\right)\right)$ (resp., $\left.I_{T_{2}-}^{\alpha}\left(L_{p^{\prime}}\left(\left(T_{1}, T_{2}\right) ; R\right)\right)\right)$ the image of $L_{p}\left(\left(T_{1}, T_{2}\right) ; R\right)$ (resp., $\left.L_{p^{\prime}}\left(\left(T_{1}, T_{2}\right) ; R\right)\right)$ by the operator $I_{T_{1}+}^{\alpha}$ (resp., $\left.I_{T_{2}-}^{\alpha}\right)$.

Suppose now that $k \in I_{T_{1}+}^{\alpha}\left(L_{p}\left(\left(T_{1}, T_{2}\right) ; R\right)\right), \zeta_{T_{2}-} \in I_{T_{2}-}^{1-\alpha}\left(L_{p^{\prime}}\left(\left(T_{1}, T_{2}\right) ; R\right)\right)$ with $1 / p+1 / p^{\prime} \leq 1$, where $\alpha p<1$, and that $k\left(T_{1}+\right)$, the right-side limit of $k$ at $T_{1}$, exists. Then following Zähle [27] we define

$$
\int_{T_{1}}^{T_{2}} k d \zeta=(-1)^{\alpha} \int_{T_{1}}^{T_{2}} D_{T_{1}+}^{\alpha} k[r] D_{T_{2}-}^{1-\alpha} \zeta_{T_{2}-}[r] d r
$$

where $\zeta_{T_{2}-}(r)=\zeta(r)-\zeta\left(T_{2}-\right)$, for $r \in\left(T_{1}, T_{2}\right)$. In fact, under the above assumptions, the appearing fractional derivatives in the integrals are well defined taking $\tilde{V}=\hat{V}=R$.

Consider now the separable Hilbert space $L_{2}(V)$ of Hilbert-Schmidt operators on $V$ with the usual norm $\|\cdot\|_{L_{2}(V)}$ and inner product $(\cdot, \cdot)_{L_{2}(V)}$. A base in this space is given by

$$
E_{i j} e_{k}=\left\{\begin{array}{lll}
0 & : & j \neq k \\
e_{i} & : & j=k
\end{array}\right.
$$

Let us consider mappings $K:\left[T_{1}, T_{2}\right] \rightarrow L_{2}(V)$ and $\omega:\left[T_{1}, T_{2}\right] \rightarrow V$ such that $k_{j i}=$ $\left(K, E_{j i}\right)_{L_{2}(V)} \in I_{T_{1}+}^{\alpha}\left(L_{p}\left(\left(T_{1}, T_{2}\right) ; R\right)\right)$ and $k_{j i}\left(T_{1}+\right)$ exists and $\alpha p<1$. Moreover, $\zeta_{i T_{2}-}=\left(\omega_{T_{2}-}(t), e_{i}\right) \in I_{T_{2}-}^{1-\alpha}\left(L_{p^{\prime}}\left(\left(T_{1}, T_{2}\right) ; R\right)\right)$, where $1 / p+1 / p^{\prime} \leq 1$. In addition,

Copyright $@$ by SIAM. Unauthorized reproduction of this article is prohibited. 


$$
\left[T_{1}, T_{2}\right] \ni r \mapsto\left\|D_{T_{1}+}^{\alpha} K[r]\right\|_{L_{2}(V)}\left|D_{T_{2}-}^{1-\alpha} \omega_{T_{2}-}[r]\right| \in L_{1}\left(\left(T_{1}, T_{2}\right) ; R\right) .
$$

We then introduce

$$
\int_{T_{1}}^{T_{2}} K d \omega:=(-1)^{\alpha} \int_{T_{1}}^{T_{2}} D_{T_{1}+}^{\alpha} K[r] D_{T_{2}-}^{1-\alpha} \omega_{T_{2}-}[r] d r
$$

Due to Pettis's theorem and the separability of $V$, the integrand is weakly measurable and hence measurable. In addition, we can present this integral by

$$
\int_{T_{1}}^{T_{2}} K d \omega=\sum_{j}\left(\sum_{i} \int_{T_{1}}^{T_{2}} D_{T_{1}+}^{\alpha} k_{j i}[r] D_{T_{2}-}^{1-\alpha} \zeta_{i T_{2}-}[r] d r\right) e_{j}
$$

with norm given by

$$
\begin{aligned}
\left|\int_{T_{1}}^{T_{2}} K d \omega\right| & =\left(\sum_{j}\left|\sum_{i} \int_{T_{1}}^{T_{2}} D_{T_{1}+}^{\alpha} k_{j i}[r] D_{T_{2}-}^{1-\alpha} \zeta_{i T_{2}-}[r] d r\right|^{2}\right)^{\frac{1}{2}} \\
& \leq \int_{T_{1}}^{T_{2}}\left\|D_{T_{1}+}^{\alpha} K[r]\right\|_{L_{2}(V)}\left|D_{T_{2}-}^{1-\alpha} \omega_{T_{2}-}[r]\right| d r .
\end{aligned}
$$

For $H>1 / 2$, in what follows we fix parameters $1 / 2<\beta<\beta^{\prime}<\beta^{\prime \prime}<H$. Under this choice, let $\Omega$ be the $\left(\theta_{t}\right)_{t \in R}$-invariant set of paths $\omega: R \rightarrow V$ which are $\beta^{\prime \prime}$-Hölder continuous on any compact subinterval of $R$ and are zero at zero. Later we will need to formulate asymptotical conditions for the set of these paths.

As the flow $\left(\theta_{t}\right)_{t \in R}$ on $\Omega$ of nonautonomous perturbations we consider

$$
\theta: R \times \Omega \rightarrow \Omega, \quad \theta_{t} \omega(\cdot)=\omega(t+\cdot)-\omega(t) .
$$

Lemma 2.4 (see [5]). Suppose that $K:\left[T_{1}, T_{2}\right] \mapsto L_{2}(V)$ is $\beta$-Hölder continuous where $1-\beta^{\prime}<\alpha<\beta$. Then the integral

$$
\int_{T_{1}}^{T_{2}} K(r) d \omega(r) \in V
$$

is well defined in the sense of (2.8). If for $0 \leq \tau \leq T_{1}$ the mapping $K:\left[T_{1}-\tau, T_{2}\right] \mapsto$ $L_{2}(V)$ is $\beta$-Hölder continuous, then

$$
\int_{T_{1}}^{T_{2}} K(r) d \omega(r)=\int_{T_{1}-\tau}^{T_{2}-\tau} K(r+\tau) d \theta_{\tau} \omega(r) .
$$

3. Evolution equations driven by a Hölder continuous function. We now consider the following evolution equation on $[0, T]$ :

$$
d u=A u d t+F(u) d t+G(u) d \omega, \quad u(0)=u_{0} \in V,
$$

driven by a Hölder continuous path $\omega$. The integral with respect to $d \omega$ is interpreted in the sense of the previous section.

In what follows we describe the different terms on the right-hand side of (3.1). Let $-A$ be a strictly positive and symmetric operator with a compact inverse which is the generator of an analytic exponential decreasing semigroup $S$ on $V$. For $\delta \geq 0$ we introduce the spaces $V_{\delta}:=D\left((-A)^{\delta}\right)$ with norm $|\cdot|_{V_{\delta}}=\left|(-A)^{\delta} \cdot\right|$ such that $V=V_{0}$.

Copyright (c) by SIAM. Unauthorized reproduction of this article is prohibited. 
The spaces $V_{\delta}, \delta>0$ are supposed to be compactly embedded in $V$. From now on, assume that $\left(e_{i}\right)_{i \in N}$ is the complete orthonormal base generated by the eigenelements of $-A$ with associated eigenvalues $\left(\lambda_{i}\right)_{i \in N}$.

Let $L\left(V_{\delta}, V_{\gamma}\right)$ denote the space of continuous linear operators from $V_{\delta}$ into $V_{\gamma}$. Then there exists a constant $c>0$ such that we have the estimates

$$
\begin{gathered}
|S(t)|_{L\left(V_{\alpha}, V_{\gamma}\right)}=\left|(-A)^{\gamma} S(t)\right|_{L\left(V_{\alpha}, V\right)} \leq \frac{c}{t^{\gamma-\alpha}} e^{-\lambda_{1} t} \quad \text { for } \gamma \geq \alpha, \quad t \in(0, T], \\
|S(t)-\mathrm{id}|_{L\left(V_{\sigma+\mu}, V_{\theta+\mu}\right)} \leq c t^{\sigma-\theta}, \quad \text { for } \theta \geq 0, \quad \sigma \in(\theta, 1+\theta], \quad \mu \in R
\end{gathered}
$$

see [6, Chapter 2]. In particular, formula (1.14) on p. 83 of that book becomes (3.2) above in bounded intervals, where we shall use it.

From these inequalities, for $\mu, \eta \in(0,1]$ and $0 \leq \delta<\gamma+\mu$ there exists a $c>0$ such that for $0 \leq q \leq r \leq s \leq t$, we can derive that

$$
\begin{aligned}
& |S(t-r)-S(t-q)|_{L\left(V_{\delta}, V_{\gamma}\right)} \leq c(r-q)^{\mu}(t-r)^{-\mu-\gamma+\delta} \\
& |S(t-r)-S(s-r)-S(t-q)+S(s-q)|_{L(V)} \leq c(t-s)^{\mu}(r-q)^{\eta}(s-r)^{-(\mu+\eta)} .
\end{aligned}
$$

As usual, $L(V)$ denotes $L(V, V)$.

Coming back to (3.1), the mapping $F: V \rightarrow V$ is supposed to be Lipschitz continuous, but we are going to assume that $F \equiv 0$, simplification that we make for the sake of brevity since the $d t$-nonlinearity is not the interesting term in system (3.1), and of course that we would achieve the same existence results as we obtain below assuming that $F$ were Lipschitz. However, for the existence of an attractor we also would need to assume that $F$ has a sufficiently small Lipschitz constant.

The nonlinear operator $G: V \rightarrow L_{2}(V)$ is assumed to be a twice continuously Fréchet-differentiable operator with bounded first and second derivatives. Let us denote, respectively, by $c_{D G}, c_{D^{2} G}$ the bounds for these derivatives and set $c_{G}=$ $\|G(0)\|_{L_{2}(V)}$. Then, for $u_{1}, u_{2}, v_{1}, v_{2} \in V$, we have

$$
\begin{aligned}
& \left\|G\left(u_{1}\right)-G\left(v_{1}\right)\right\|_{L_{2}(V)} \leq c_{D G}\left|u_{1}-v_{1}\right| \\
& \left\|G\left(u_{1}\right)\right\|_{L_{2}(V)} \leq c_{G}+c_{D G}\left|u_{1}\right| \\
& \quad\left\|G\left(u_{1}\right)-G\left(v_{1}\right)-\left(G\left(u_{2}\right)-G\left(v_{2}\right)\right)\right\|_{L_{2}(V)} \\
& \quad \leq c_{D G}\left|u_{1}-v_{1}-\left(u_{2}-v_{2}\right)\right|+c_{D^{2} G}\left|u_{1}-u_{2}\right|\left(\left|u_{1}-v_{1}\right|+\left|u_{2}-v_{2}\right|\right) .
\end{aligned}
$$

(The proof of the last inequality can be found in [20].)

Finally, the driving function $\omega \in C^{\beta^{\prime}}([0, T] ; V)$ for $1 / 2<\beta<\beta^{\prime}<H$ and $1-\beta^{\prime}<\alpha<\beta$. We are then interested in solving (3.1) in a mild sense, which means that for $t \in[0, T]$ we have to solve

$$
u(t)=S(t) u_{0}+\int_{0}^{t} S(t-r) G(u(r)) d \omega .
$$

According to the definition of the integral given in the previous section, we need to estimate the fractional derivative of the term $S(t-\cdot) G(u(\cdot))$.

LEMma 3.1 (see [5]). Let $S$ be the semigroup generated by $-A$, assume that $G$ is Lipschitz and $u \in C^{\beta, \sim}\left(\left[T_{1}, T_{2}\right] ; V\right)$. Then for the orthonormal base $\left(e_{i}\right)_{i \in N}$ of $V$, for any $i, j \in N$,

$$
\left(e_{i}, S(t-\cdot) G(u(\cdot)) e_{j}\right) \in I_{T_{1}+}^{\alpha}\left(L_{p}\left(\left(T_{1}, T_{2}\right) ; R\right)\right)
$$

Copyright (C) by SIAM. Unauthorized reproduction of this article is prohibited. 
if $\alpha p<1$. In addition, the mapping $r \mapsto D_{T_{1}+}^{\alpha} S(t-\cdot) G(u(\cdot))[r]$ is measurable on $\left[T_{1}, t\right]$ for $t \leq T_{2}$ and satisfies the estimate

$$
\left\|D_{T_{1}+}^{\alpha} S(t-\cdot) G(u(\cdot))[r]\right\|_{L_{2}(V)} \leq c\left(1+\|u\|_{\beta, \sim}\right)\left(r-T_{1}\right)^{-\alpha}\left(1+\frac{\left(r-T_{1}\right)^{\beta}}{(t-r)^{\beta}}\right) .
$$

Similarly, assuming that $u \in C^{\beta}\left(\left[T_{1}, T_{2}\right] ; V\right)$, then

$$
\left\|D_{T_{1}+}^{\alpha} S(t-\cdot) G(u(\cdot))[r]\right\|_{L_{2}(V)} \leq c\left(1+\|u\|_{\beta}\right)\left(r-T_{1}\right)^{-\alpha}\left(1+\left(r-T_{1}\right)^{\beta}+\frac{\left(r-T_{1}\right)^{\beta}}{(t-r)^{\beta}}\right) .
$$

In addition, $\left(\omega_{T_{2}-}(t), e_{i}\right) \in I_{T_{2}-}^{1-\alpha}\left(L_{p^{\prime}}\left(\left(T_{1}, T_{2}\right) ; R\right)\right)$ for any $p^{\prime}>1$ and $\omega \in C^{\beta^{\prime}}\left(\left[T_{1}, T_{2}\right] ; V\right)$ for $\beta^{\prime}>1-\alpha$, and

$$
\left|D_{T_{2}-}^{1-\alpha} \omega_{T_{2}-}[r]\right| \leq c|||\omega| \|_{\beta^{\prime}, T_{1}, T_{2}}\left(T_{2}-r\right)^{\beta^{\prime}+\alpha-1} .
$$

Existence of solutions of (3.4) has been investigated in [20] and [8] when considering as integrators of the integrals a fractional Brownian motion with Hurst parameter in $(1 / 2,1)$ and certain phase spaces, which are not as natural as the space of Hölder continuous functions. In this article we present the existence theory in the space of Hölder continuous solutions for appropriate exponents. More precisely, we have the following existence result, whose proof can be found in [5].

Theorem 3.2 (see [5]). If $u_{0} \in V$, then for every $T>0$, (3.4) has a unique solution $u$ in $C^{\beta, \sim}([0, T] ; V)$.

If $u_{0} \in V_{\beta}$, then for every $T>0,(3.4)$ has a unique solution $u$ in $C^{\beta}([0, T] ; V)$.

The main reason to consider the space $C^{\beta, \sim}([0, T] ; V)$ is that $t \mapsto S(t) u_{0}$ is not a $\beta$-Hölder continuous function but an element of that space. However, if $u_{0} \in V_{\beta}$, then $t \mapsto S(t) u_{0}$ is an element of $C^{\beta}([0, T] ; V)$. Considering the equivalent norm from (2.5) on $C^{\beta, \sim}([0, T] ; V)$, we are able to adapt $\rho$ to the data of our problem (in particular to $T$ ) such that the right-hand side of (3.4) forms an operator which satisfies the conditions of the Banach fixed point theorem if $u_{0} \in V$. In particular (see [5]), we obtain estimates of the integral like

$$
\begin{gathered}
\left\|\int_{0}^{\cdot} S(\cdot-r) G(u(r)) d \omega\right\|_{\beta, \sim, 0, T} \leq c_{T}\|\omega\|_{\beta^{\prime}, 0, T}\left(1+\|u\|_{\beta, \sim, 0, T}\right), \\
\left\|\int_{0} S(\cdot-r) G(u(r)) d \omega\right\|_{\beta, 0, T} \leq c_{T}\|\omega\|_{\beta^{\prime}, 0, T}\left(1+\|u\|_{\beta, 0, T}\right) .
\end{gathered}
$$

Assuming that $u_{0} \in V$, the corresponding solution $u$ of (3.4) satisfies $u(T) \in V_{\beta}$ for every $T>0$, due to

$$
|u(T)|_{V_{\beta}} \leq c T^{-\beta}\left|u_{0}\right|+c_{T}|||\omega| \|_{\beta^{\prime}, 0, T}\left(1+\|u\|_{\beta, \sim, 0, T}\right)<\infty .
$$

The constant $c_{T}$ in the above formulas depends on the operators $S, G$ and is bounded when $T$ is bounded. $c_{T}$ can be chosen independently of $\omega$.

In addition to the previous estimates we also have the following one.

Lemma 3.3. For $1-\beta^{\prime}<\alpha<\beta$ and $u \in C^{\beta}([0, T] ; V)$,

$$
\left|\int_{0}^{T} S(T-r) G(u(r)) d \omega\right|_{V_{\beta}} \leq c_{T}\left|\left\|\omega|\||_{\beta^{\prime}, 0, T}\left(1+\|u\|_{\beta, 0, T}\right) .\right.\right.
$$

Copyright $@$ by SIAM. Unauthorized reproduction of this article is prohibited. 
Proof. Take $1>\alpha^{\prime}>\alpha$ such that $\alpha^{\prime}+\beta<\alpha+\beta^{\prime}$; then the following inequalities hold:

$$
\begin{aligned}
&\left|(-A)^{\beta} \int_{0}^{T} S(T-r) G(u(r)) d \omega\right| \\
& \leq \frac{1}{\Gamma(1-\alpha)} \int_{0}^{T}\left(\frac{\left|S(T-r)(-A)^{\beta} G(u(r))\right|}{r^{\alpha}}\right. \\
&+\alpha \int_{0}^{r} \frac{\left|(S(T-r)-S(T-q))(-A)^{\beta} G(u(r))\right|}{(r-q)^{1+\alpha}} d q \\
&\left.+\alpha \int_{0}^{r} \frac{\left|S(T-q)(-A)^{\beta}(G(u(r))-G(u(q)))\right|}{(r-q)^{1+\alpha}} d q\right)\left|\|\omega \mid\|_{\beta^{\prime}, 0, T}(T-r)^{\alpha+\beta^{\prime}-1} d r\right. \\
& \leq c\left|\left\|\omega | \| | _ { \beta ^ { \prime } , 0 , T } ( 1 + \| u \| _ { \beta , 0 , T } ) \int _ { 0 } ^ { T } \left(\frac{1}{r^{\alpha}(T-r)^{\beta}}\right.\right.\right. \\
&\left.+\int_{0}^{r} \frac{(r-q)^{\alpha^{\prime}}}{(T-r)^{\beta+\alpha^{\prime}}(r-q)^{1+\alpha}} d q+\int_{0}^{r} \frac{(r-q)^{\beta}}{(T-r)^{\beta}(r-q)^{1+\alpha}} d q\right)(T-r)^{\alpha+\beta^{\prime}-1} d r \\
& \leq c_{T}\|\omega \mid\| \|_{\beta^{\prime}, 0, T}\left(1+\|u\|_{\beta, 0, T}\right),
\end{aligned}
$$

where the last inequality is true due to the choice made for the parameters.

3.1. Nonautonomous dynamical system associated to (3.4). According to Lemmas 2.4 and 3.1, for $t, \tau \in R^{+}$yields

$$
\int_{\tau}^{t+\tau} S(t+\tau-r) G(u(r)) d \omega(r)=\int_{0}^{t} S(t-r) G(u(r+\tau)) d \theta_{\tau} \omega(r),
$$

where $S$ and $G$ satisfy the corresponding conditions of Lemma 3.1. From this formula it follows easily that the solution of (3.4) generates a nonautonomous dynamical system $\varphi$ on $R^{+}$with state space $V$ :

$$
\begin{aligned}
& \varphi: R^{+} \times \Omega \times V \rightarrow V, \\
& \varphi\left(t, \omega, u_{0}\right)=S(t) u_{0}+\int_{0}^{t} S(t-r) G(u(r)) d \omega .
\end{aligned}
$$

However, later on from $\varphi$ we shall derive another nonautonomous dynamical system but with discrete time set $Z^{+}$.

Next we introduce some stopping times which are generated by elements of $\Omega$. These stopping times are needed to keep $\||\omega|\|_{\beta^{\prime}}$ small, which is necessary to obtain appropriate a priori estimates for the solution.

In a first step, fix $\mu>0$ and define the stopping times as follows:

$$
\begin{aligned}
& T(\omega)=\inf \left\{\tau>0:|||\omega| \|_{\beta^{\prime}, 0, \tau}+\mu \tau^{1-\beta^{\prime}} \geq \mu\right\}, \\
& \hat{T}(\omega)=\sup \left\{\tau<0:|||\omega|||_{\beta^{\prime}, \tau, 0}+\mu|\tau|^{1-\beta^{\prime}} \geq \mu\right\} .
\end{aligned}
$$

Lemma 3.4. For $\omega \in \Omega$ we have that $T(\omega),-\hat{T}(\omega)$ are in $(0,1]$. In addition, $T(\omega)=-\hat{T}\left(\theta_{T(\omega)} \omega\right)$ and $\hat{T}(\omega)=-T\left(\theta_{\hat{T}(\omega)} \omega\right)$.

Proof. It is easily seen that $|T(\omega)|,|\hat{T}(\omega)| \leq 1$. Moreover, since $\omega$ has a finite $\beta^{\prime \prime}$-Hölder seminorm and $\beta^{\prime}<\beta^{\prime \prime}$ we have

$$
\lim _{\tau \downarrow 0}|||\omega|||_{\beta^{\prime}, 0, \tau}=0
$$

Copyright $@$ by SIAM. Unauthorized reproduction of this article is prohibited. 
and, in addition,

$$
\lim _{\tau \rightarrow+\infty}|||\omega| \|_{\beta^{\prime}, 0, \tau}+\mu \tau^{1-\beta^{\prime}}=+\infty
$$

where we suppose that this condition holds for any $\omega \in \Omega$.

Therefore, thanks to the intermediate value theorem, we only need to prove the continuity of the strictly increasing mapping $\tau \rightarrow\|\| \omega \|_{\beta^{\prime}, 0, \tau}+\mu \tau^{1-\beta^{\prime}}$ to ensure that there is a time $\hat{\tau}_{0}$ such that $\||\omega|\|_{\beta, 0, \hat{\tau}_{0}}+\mu \hat{\tau}_{0}^{1-\beta^{\prime}}=\mu$, which means that we can replace the inequality of (3.9) by an equality.

Fixed $\tau_{0}>0$ and define $\omega^{\tau_{0}}$ given by

$$
\omega^{\tau_{0}}(s)= \begin{cases}\omega(s) & : \quad \text { for } s<\tau_{0} \\ \omega\left(\tau_{0}\right) & : \quad \text { for } s \geq \tau_{0}\end{cases}
$$

Thus, for $\tau \geq \tau_{0}$,

$\left.|||\omega|\right|_{\beta^{\prime}, 0, \tau}-|||\omega|||_{\beta^{\prime}, 0, \tau_{0}}=|||\omega|||_{\beta^{\prime}, 0, \tau}-||\left|\omega^{\tau_{0}}\right|||_{\beta^{\prime}, 0, \tau} \leq||\left|\omega-\omega^{\tau_{0}}\right|||_{\beta^{\prime}, 0, \tau}=\|\left.|| \omega||\right|_{\beta^{\prime}, \tau_{0}, \tau}$,

and because

$$
\lim _{\tau \downarrow \tau_{0}}\left|\left\|\omega \left|\left\|\left.\right|_{\beta^{\prime}, \tau_{0}, \tau} \leq \lim _{\tau \downarrow \tau_{0}}\left|\left\|\omega \left|\left\|\left.\right|_{\beta^{\prime \prime}, \tau_{0}, \tau}\left(\tau-\tau_{0}\right)^{\beta^{\prime \prime}-\beta^{\prime}} \leq \lim _{\tau \downarrow \tau_{0}}\left|\|\omega \mid\|_{\beta^{\prime \prime}, 0, \tau}\left(\tau-\tau_{0}\right)^{\beta^{\prime \prime}-\beta^{\prime}}=0,\right.\right.\right.\right.\right.\right.\right.\right.\right.
$$

then the mentioned continuity property is true. Note that we could obtain a similar conclusion when taking $\tau \uparrow \tau_{0}$. Moreover, because $T \mapsto\||\omega|\|_{\beta^{\prime}, 0, T}+\mu T^{1-\beta^{\prime}}$ is strictly increasing and $T \mapsto|||\omega|||_{\beta^{\prime}, T, 0}+\mu|T|^{1-\beta^{\prime}}$ is strictly decreasing, it is easily seen that

$$
\mu=\left\||| \omega\left|\left\|_{\beta^{\prime}, 0, T(\omega)}+\mu T(\omega)^{1-\beta^{\prime}}=\right\|\right| \mid \theta_{T(\omega)} \omega\right\|_{\beta^{\prime}, \hat{T}\left(\theta_{T(\omega)} \omega\right), 0}+\mu\left(-\hat{T}\left(\theta_{T(\omega)} \omega\right)\right)^{1-\beta^{\prime}},
$$

which is possible only if $T(\omega)=-\hat{T}\left(\theta_{T(\omega)} \omega\right)$.

From $T(\omega), \hat{T}(\omega)$ we derive a sequence of stopping times. For $\omega \in \Omega$ we define

$$
T_{i}(\omega)=\left\{\begin{array}{lcc}
0 & : & i=0, \\
T_{i-1}(\omega)+T\left(\theta_{T_{i-1}(\omega)} \omega\right) & : & i \in N, \\
T_{i+1}(\omega)+\hat{T}\left(\theta_{T_{i+1}(\omega)} \omega\right) & : & i \in-N .
\end{array}\right.
$$

Then $\left(T_{i}(\omega)\right)_{i \in Z}$ satisfies the cocycle property: for $i, j \in Z$ we have

$$
T_{0}(\omega)=0, \quad T_{i}(\omega)+T_{j}\left(\theta_{T_{i}(\omega)} \omega\right)=T_{i+j}(\omega) .
$$

Note that with the previous notation we are identifying $T(\omega)$ with $T_{1}(\omega)$ and $\hat{T}(\omega)$ with $T_{-1}(\omega)$.

Lemma 3.5. Let $t_{1} \leq t_{2}$. Then

$$
t_{1}+\hat{T}\left(\theta_{t_{1}} \omega\right) \leq t_{2}+\hat{T}\left(\theta_{t_{2}} \omega\right) .
$$

Proof. Suppose that the contrary inequality holds, which means that $\hat{T}\left(\theta_{t_{1}} \omega\right)>$ $\hat{T}\left(\theta_{t_{2}} \omega\right)$. Therefore, $\left(-\hat{T}\left(\theta_{t_{1}} \omega\right)\right)^{1-\beta^{\prime}}<\left(-\hat{T}\left(\theta_{t_{2}} \omega\right)\right)^{1-\beta^{\prime}}$ and moreover

$$
\begin{aligned}
\mu & =||\left|\theta_{t_{2}} \omega\right| \|_{\beta^{\prime}, \hat{T}\left(\theta_{t_{2}} \omega\right), 0}+\mu\left(-\hat{T}\left(\theta_{t_{2}} \omega\right)\right)^{1-\beta^{\prime}} \\
& =\sup _{\hat{T}\left(\theta_{t_{2}} \omega\right)+t_{2} \leq s<t \leq t_{2}} \frac{|\omega(t)-\omega(s)|}{|t-s|^{\beta^{\prime}}}+\mu\left(-\hat{T}\left(\theta_{t_{2}} \omega\right)\right)^{1-\beta^{\prime}} \\
& >\sup _{\hat{T}\left(\theta_{t_{1}} \omega\right)+t_{1} \leq s<t \leq t_{1}} \frac{|\omega(t)-\omega(s)|}{|t-s|^{\beta^{\prime}}}+\mu\left(-\hat{T}\left(\theta_{t_{1}} \omega\right)\right)^{1-\beta^{\prime}} \\
& =||\left|\theta_{t_{1}} \omega\right|||_{\beta^{\prime}, \hat{T}\left(\theta_{t_{1}} \omega\right), 0}+\mu\left(-\hat{T}\left(\theta_{t_{1}} \omega\right)\right)^{1-\beta^{\prime}}=\mu .
\end{aligned}
$$

However, this chain of inequalities causes a contradiction. 
If $t_{2}+\hat{T}\left(\theta_{t_{2}} \omega\right) \leq t_{1} \leq t_{2}$, then iterating the formula in the previous lemma we obtain that the stopping times enjoy the following order property:

$$
\begin{aligned}
\cdots & \leq t_{1}+\hat{T}\left(\theta_{t_{1}} \omega\right)+\hat{T}\left(\theta_{t_{1}+\hat{T}\left(\theta_{t_{1}} \omega\right)} \omega\right) \\
& \leq t_{2}+\hat{T}\left(\theta_{t_{2}} \omega\right)+\hat{T}\left(\theta_{t_{2}+\hat{T}\left(\theta_{t_{2}} \omega\right)} \omega\right) \\
& \leq t_{1}+\hat{T}\left(\theta_{t_{1}} \omega\right) \leq t_{2}+\hat{T}\left(\theta_{t_{2}} \omega\right) \leq t_{1} \leq t_{2} .
\end{aligned}
$$

3.2. Global attractor for the nonautonomous dynamical system associated to (3.4). As a preparation of the next key result, Lemma 3.7 below, we formulate the following Gronwall-like lemma.

Lemma 3.6. Let $\lambda, v_{0}, k_{0}, k_{1}<1, k_{2}$ be positive numbers and let $\left(t_{i}\right)_{i \in Z^{+}}$be a sequence of positive numbers, with $t_{0}=0$, such that

$$
t_{i-1}-t_{i-2} \leq-\frac{2}{\lambda} \log k_{1} \quad \text { for } i \geq 2 .
$$

Suppose that for a sequence of positive numbers $\left(U_{i}\right)_{i \in N}$ the following inequalities hold true:

$$
\begin{aligned}
U_{i} \leq & k_{0} v_{0} e^{-\lambda t_{i-1}}+\sum_{m=1}^{i-1} k_{1} U_{m} e^{-\lambda\left(t_{i-1}-t_{m}\right)} \\
& +\sum_{m=1}^{i-1} e^{-\lambda\left(t_{i-1}-t_{m}\right)} k_{2}+k_{2}, \quad i=1,2,3, \ldots
\end{aligned}
$$

Then we have ${ }^{1}$

$$
\begin{aligned}
U_{i} \leq & \left(k_{0} v_{0}+k_{2}\right)\left(1+k_{1}\right)^{i-1} e^{-\frac{\lambda}{2} t_{i-1}} \\
& +\sum_{m=1}^{i-1} 2 k_{2}\left(1+k_{1}\right)^{i-1-m} e^{-\frac{\lambda}{2}\left(t_{i-1}-t_{m}\right)} \quad \text { for } i=1,2,3, \ldots
\end{aligned}
$$

Proof. First, note that the inequality

$$
k_{1}+e^{-x} \leq\left(1+k_{1}\right) e^{-\frac{x}{2}}
$$

holds true for $x \in\left[0,-2 \log k_{1}\right]$.

Denote the right-hand side of (3.13) by $Z_{i}$ and the right-hand side of (3.14) by $S_{i}$. We want to prove that $U_{i} \leq S_{i}$ for all $i \geq 1$, for which it is enough to prove by induction that $Z_{i} \leq S_{i}$.

For $i=1$, from (3.13) we have $U_{1} \leq Z_{1}=k_{0} v_{0}+k_{2}$. From (3.14) we have $S_{1}=k_{0} v_{0}+k_{2}$, so $Z_{1}=S_{1}$.

For $i=2$, from (3.13) we have $Z_{2}=k_{0} v_{0} e^{-\lambda t_{1}}+k_{1} U_{1}+2 k_{2}$. From (3.14) we have $S_{2}=\left(k_{0} v_{0}+k_{2}\right)\left(1+k_{1}\right) e^{-\frac{\lambda}{2} t_{1}}+2 k_{2}$. It suffices to take into account that from (3.15) we have

$$
k_{1}+e^{-\lambda t_{1}} \leq\left(1+k_{1}\right) e^{-\frac{\lambda}{2} t_{1}}
$$

\footnotetext{
${ }^{1}$ The sum $\sum_{m=1}^{i-1}$ is assumed to be zero for $i=1$.
}

Copyright $@$ by SIAM. Unauthorized reproduction of this article is prohibited. 
since by (3.12) in particular $\lambda t_{1} \leq-2 \log k_{1}$, Therefore,

$$
\begin{aligned}
Z_{2} & =k_{0} v_{0} e^{-\lambda t_{1}}+k_{1} U_{1}+2 k_{2} \leq\left(k_{0} v_{0}+k_{2}\right) e^{-\lambda t_{1}}+k_{1}\left(k_{0} v_{0}+k_{2}\right)+2 k_{2} \\
& \leq\left(k_{0} v_{0}+k_{2}\right)\left(1+k_{1}\right) e^{-\frac{\lambda}{2} t_{1}}+2 k_{2}=S_{2} .
\end{aligned}
$$

For $i \geq 3$ we apply induction. First note that

$$
e^{-\lambda\left(t_{i-1}-t_{i-2}\right)} Z_{i-1}=Z_{i}-k_{1} U_{i-1}-2 k_{2}+k_{2} e^{-\lambda\left(t_{i-1}-t_{i-2}\right)}
$$

and thereby, assuming that $Z_{i-1} \leq S_{i-1}$, thanks to (3.12) and (3.15),

$$
\begin{aligned}
U_{i} & \leq Z_{i} \leq e^{-\lambda\left(t_{i-1}-t_{i-2}\right)} Z_{i-1}+k_{1} U_{i-1}+2 k_{2} \leq\left(e^{-\lambda\left(t_{i-1}-t_{i-2}\right)}+k_{1}\right) Z_{i-1}+2 k_{2} \\
& \leq\left(e^{-\lambda\left(t_{i-1}-t_{i-2}\right)}+k_{1}\right) S_{i-1}+2 k_{2} \leq\left(1+k_{1}\right) e^{-\frac{\lambda}{2}\left(t_{i-1}-t_{i-2}\right)} S_{i-1}+2 k_{2}=S_{i} .
\end{aligned}
$$

Next we obtain an appropriate a priori estimate for the solution of our equation when assuming that the initial condition is regular, namely, $u_{0} \in V_{\beta}$. Later on this a priori estimate will be the key to obtain an absorbing set, the main ingredient to ensure the existence of a pullback attractor for the system.

In what follows, $c$ shall denote a positive constant whose value is unimportant and can of course change from line to line, and may depend on $S$ and $G$ but not on $\omega$.

Lemma 3.7. For $\omega \in \Omega$ let $u$ be a solution of (3.1) where $u_{0} \in V_{\beta}$ and let $\left(T_{i}\left(\theta_{T_{j}(\omega)} \omega\right)\right)_{i \in Z}$ be the sequence of stopping times defined by (3.10). Then we have that

$$
\begin{aligned}
\|u\|_{\beta, T_{i-1}\left(\theta_{T_{j}} \omega\right), T_{i}\left(\theta_{T_{j}} \omega\right) \leq} & c e^{-\lambda_{1} T_{i-1}\left(\theta_{T_{j}} \omega\right)}\left|u_{0}\right|_{V_{\beta}}+c \mu\|u\|_{\beta, T_{i-1}\left(\theta_{T_{j}} \omega\right), T_{i}\left(\theta_{T_{j}} \omega\right)} \\
& +\sum_{m=1}^{i-1} c \mu e^{-\lambda_{1}\left(T_{i-1}\left(\theta_{T_{j}} \omega\right)-T_{m}\left(\theta_{T_{j}} \omega\right)\right)}\|u\|_{\beta, T_{m-1}\left(\theta_{T_{j}} \omega\right), T_{m}\left(\theta_{T_{j}} \omega\right)} \\
& +\sum_{m=1}^{i-1} e^{-\lambda_{1}\left(T_{i-1}\left(\theta_{T_{j}} \omega\right)-T_{m}\left(\theta_{T_{j}} \omega\right)\right)} c \mu+c \mu,
\end{aligned}
$$

where $T_{j}=T_{j}(\omega)$, and $\lambda_{1}$ is the smallest eigenvalue of $-A$.

The proof of this lemma can be found in the appendix.

The cocycle property of $\left(T_{i}(\omega)\right)_{i \in Z}$ allows us to introduce a discrete nonautonomous dynamical system $\Phi$ on $V$ with time set $T^{+}=Z^{+}$for every $\omega \in \Omega$. In particular, we consider the new shift given by

$$
\tilde{\theta}: Z \times Z \rightarrow Z
$$

defined by $\tilde{\theta}_{i} j=i+j$, for $i, j \in Z$. Then we define

$$
\Phi: Z^{+} \times Z \times \Omega \times V \rightarrow V
$$

as

$$
\begin{aligned}
\Phi\left(i, j, \omega, u_{0}\right) & =S\left(T_{i}\left(\theta_{T_{j}(\omega)} \omega\right)\right) u_{0}+\int_{0}^{T_{i}\left(\theta_{T_{j}(\omega)} \omega\right)} S\left(T_{i}\left(\theta_{T_{j}(\omega)} \omega\right)-r\right) G(u(r)) d \theta_{T_{j}(\omega)} \omega \\
& =\varphi\left(T_{i}\left(\theta_{T_{j}(\omega)} \omega\right), \theta_{T_{j}(\omega)} \omega, u_{0}\right) .
\end{aligned}
$$

Note that $\Phi\left(i, j, \omega, u_{0}\right)$ is given by the solution $u$ of (3.4) for the noise path $\theta_{T_{j}(\omega)} \omega$ at time $T_{i}\left(\theta_{T_{j}(\omega)} \omega\right)$. We would like to emphasize that, in the definition of $\Phi, \omega$ only acts as a parameter. 
We now specify the constant $\mu$. Let $c>0$ be the constant from Lemma 3.7. We choose $\mu$ sufficiently small such that for $k_{1}(\mu)=c \mu /(1-c \mu)<1$ the following inequality holds:

$$
1<-\frac{2}{\lambda_{1}} \log k_{1}(\mu)
$$

Let us also define

$$
\lambda=\lambda_{1}, k_{0}=\frac{c}{1-c \mu}, k_{2}=k_{1}=k_{1}(\mu), t_{i}=T_{i}\left(\theta_{T_{j}(\omega)} \omega\right) .
$$

As we will show in Corollary 3.8 below, the choice done in (3.16) ensures the condition (3.12), and with it we will prove the existence of an absorbing ball for $\Phi$ (see Lemma 3.9).

COROLlary 3.8. Let $u_{0} \in V_{\beta}$ and suppose that $\mu$ is chosen such that (3.16) is satisfied. Then the following inequality holds true: ${ }^{2}$

$$
\begin{aligned}
\left|\Phi\left(i, j, \omega, u_{0}\right)\right| \leq & \left(1+k_{1}\right)^{i-1} e^{-\frac{\lambda_{1}}{2} T_{i-1}\left(\theta_{T_{j}(\omega)} \omega\right)}\left(k_{0}\left|u_{0}\right|_{V_{\beta}}+k_{2}\right) \\
& +\sum_{m=1}^{i-1} 2 k_{2}\left(1+k_{1}\right)^{i-1-m} e^{-\frac{\lambda_{1}}{2}\left(T_{i-1}\left(\theta_{T_{j}}(\omega) \omega\right)-T_{m}\left(\theta_{T_{j}}(\omega) \omega\right)\right)} .
\end{aligned}
$$

Proof. We only need to take into account that, by definition,

$$
\left|\Phi\left(i, j, \omega, u_{0}\right)\right|=\mid u\left(T_{i}\left(\theta_{T_{j}(\omega)} \omega\right) \mid \leq\|u\|_{\beta, T_{i-1}\left(\theta_{T_{j}(\omega)} \omega\right), T_{i}\left(\theta_{T_{j}(\omega)} \omega\right)}\right.
$$

and therefore, thanks to Lemma 3.7, we obtain the desired estimate due to the above choice of $k_{0}, k_{1}, k_{2}, T_{i}$ if in addition we take $v_{0}=\left|u_{0}\right|_{V_{\beta}}$. We would like to stress that we can apply the Gronwall-like Lemma 3.6 in this situation since the stopping times satisfy for sufficiently small $\mu$ the condition (3.12). Actually, the cocycle property and (3.16) imply

$t_{i-1}-t_{i-2}=T_{i-1}\left(\theta_{T_{j}(\omega)} \omega\right)-T_{i-2}\left(\theta_{T_{j}(\omega)} \omega\right)=T_{1}\left(\theta_{T_{i-2+j}(\omega)} \omega\right) \leq 1<-\frac{2}{\lambda_{1}} \log k_{1}(\mu)$.

Now we formulate a smallness condition for all $\omega \in \Omega$. We assume that the stopping times satisfy

$$
1>\liminf _{i \rightarrow-\infty} \frac{\left|T_{i}(\omega)\right|}{|i|} \geq d \geq \frac{2\left(\log \left(1+k_{1}(\mu)\right)+\nu\right)}{\lambda_{1}},
$$

where $\nu \in\left[0, \frac{d \lambda_{1}}{2}\right)$ is the parameter describing backward $\nu$-exponentially growing sets $\mathcal{D}_{Z, V}^{\nu}$. In addition assume that

$$
\nu+d>1
$$

We also assume that the sequence $\left(\left|T\left(\theta_{T_{i}(\omega)} \omega\right)\right|^{-\beta}\right)_{i \in Z}$ is subexponential growing for $\omega \in \Omega$ :

$$
\lim _{i \rightarrow-\infty} \frac{\log ^{+}\left(\left|T\left(\theta_{T_{i}(\omega)} \omega\right)\right|^{-\beta}\right)}{|i|}=0
$$

\footnotetext{
${ }^{2}$ Again, the sum $\sum_{m=1}^{i-1}$ is assumed to be zero for $i=1$.
} 
Later on, in section 4, we will give an example of a set $\Omega$ of $\omega$ fulfilling conditions (3.18), (3.19), and (3.20) that is $\left(\theta_{t}\right)_{t \in R^{-} \text {-invariant. }}$

We now consider the discrete nonautonomous dynamical system $\Phi$ with the set of nonautonomous perturbations $Z$ and shifts $\tilde{\theta}_{i} j=i+j$. Recall that the set system $\mathcal{D}_{Z, V}^{\nu}$ is given by the family of sets $(D(i))_{i \in Z}$ such that $D(i) \subset V$ is included in a ball with center zero and radius $r(i)$, where

$$
\limsup _{i \rightarrow-\infty} \frac{\log ^{+} r(i)}{|i|}<\nu
$$

Our next aim is to prove that the discrete nonautonomous dynamical system $\Phi$ has an absorbing set consisting in a ball $B$ contained in $\mathcal{D}_{Z, V}^{\nu}$, which means that in particular $B$ is in $V$.

Lemma 3.9. Suppose that (3.16), (3.18), (3.19), and (3.20) hold. Then $\Phi$ has a $\mathcal{D}_{Z, V^{-}}^{\nu}$ absorbing set $B(\omega)=(B(i, \omega))_{i \in Z}$, where $B(i, \omega)$ is given by a ball in $V$ with center 0 and radius

$$
R(i, \omega)=2 \sum_{m=-\infty}^{0} 2 k_{2}\left(1+k_{1}\right)^{-m} e^{\frac{\lambda_{1}}{2} T_{m}\left(\theta_{T_{i-1}(\omega)} \omega\right)} .
$$

Proof. We want to get a $\mathcal{D}_{Z, V}^{\nu}$-absorbing set $B$ for $\Phi$. In Corollary 3.8 we have obtained estimates for $\Phi$ when the initial condition $u_{0} \in V_{\beta}$, so in this proof in a first step we have to ensure that picking any $D \in \mathcal{D}_{Z, V}^{\nu}$ we can build an appropriate set $F$ depending on $D$ such that $F \in \mathcal{D}_{Z, V_{\beta}}^{\nu}$. This property will be the key to later proving that $B$ is an absorbing set for $\Phi$.

Define for $D \in \mathcal{D}_{Z, V}^{\nu}$ the set

$$
F(j, \omega):=\overline{\Phi(1, j-1, \omega, D(j-1))} .
$$

Such an $F$ is a backward $\nu$-exponentially growing set in $V_{\beta}$, since if $v_{0} \in D(j-1)$ we know that

$$
\begin{aligned}
\Phi\left(1, j-1, \omega, v_{0}\right)= & u\left(T_{1}\left(\theta_{T_{j-1}(\omega)} \omega\right)\right)=S\left(T_{1}\left(\theta_{T_{j-1}(\omega)} \omega\right)\right) v_{0} \\
& +\int_{0}^{T_{1}\left(\theta_{T_{j-1}(\omega)} \omega\right)} S\left(T_{1}\left(\theta_{T_{j-1}(\omega)} \omega\right)-r\right) G(u(r)) d \theta_{T_{j-1}(\omega)} \omega
\end{aligned}
$$

From (3.7),

$$
\begin{aligned}
\left|\Phi\left(1, j-1, \omega, v_{0}\right)\right|_{V_{\beta}} \leq & \frac{c}{\left|T_{1}\left(\theta_{T_{j-1}(\omega)} \omega\right)\right|^{\beta}}\left|v_{0}\right| \\
& +c|||\omega| \|_{\beta^{\prime}, 0, T_{1}\left(\theta_{T_{j-1}(\omega)} \omega\right)}\left(1+\|u\|_{\beta, \sim, 0, T_{1}\left(\theta_{T_{j-1}(\omega)} \omega\right)}\right) .
\end{aligned}
$$

The last term in the previous expression can be estimated by the technique of Lemma 3.7 for $i=1$ but using the $\|\cdot\|_{\beta, \sim, 0, T_{1}\left(\theta_{T_{j-1}(\omega)} \omega\right)}$-norm together with (3.5), getting that

$$
(1-c \mu)\|u\|_{\beta, \sim 0, T_{1}\left(\theta_{T_{j-1}(\omega)} \omega\right)} \leq c\left|v_{0}\right|+c \mu
$$

and therefore

$$
\begin{aligned}
& \sup _{v_{0} \in D(j-1)}\left|\Phi\left(1, j-1, \omega, v_{0}\right)\right|_{V_{\beta}} \\
& \leq\left(\frac{c}{\left|T_{1}\left(\theta_{T_{j-1}(\omega)} \omega\right)\right|^{\beta}}+\frac{c \mu}{1-c \mu}\right) \sup _{v_{0} \in D(j-1)}\left|v_{0}\right|+c \mu\left(1+\frac{c \mu}{1-c \mu}\right) .
\end{aligned}
$$

Copyright (c) by SIAM. Unauthorized reproduction of this article is prohibited. 
The first term on the right-hand side is backward $\nu$-exponentially growing, which follows from the assumption that $\left(\left|T\left(\theta_{T_{i}(\omega)} \omega\right)\right|^{-\beta}\right)_{i \in Z}$ is subexponentially growing. Indeed it is a product of two terms where one factor satisfies (2.2) while the other is $\nu$-exponentially growing.

Therefore, for any $D \in \mathcal{D}_{Z, V}^{\nu}$ the set $F(j, \omega) \in \mathcal{D}_{Z, V_{\beta}}^{\nu}$ and then there is a sequence $\left(B_{V_{\beta}}(0, \rho(i, \omega))\right)_{i \in Z}$ backward $\nu$-exponentially growing such that these balls in $V_{\beta}$ with center zero and radius $\rho(i, \omega)$ contain the sets $F(i, \omega)$. Moreover, from Corollary 3.8 we immediately have that

$$
\begin{aligned}
& \sup _{u_{0} \in F(j-i, \omega)}\left|\Phi\left(i, j-i, \omega, u_{0}\right)\right| \leq\left(1+k_{1}\right)^{i-1} e^{-\frac{\lambda_{1}}{2} T_{i-1}\left(\theta_{T_{j-i}}(\omega) \omega\right)}\left(k_{0} \rho(j-i, \omega)+k_{2}\right) \\
& +\sum_{m=1}^{i-1} 2 k_{2}\left(1+k_{1}\right)^{i-1-m} e^{-\frac{\lambda_{1}}{2}\left(T_{i-1}\left(\theta_{T_{j-i}}(\omega) \omega\right)-T_{m}\left(\theta_{T_{j-i}(\omega)} \omega\right)\right)} .
\end{aligned}
$$

Taking into account that the stopping times satisfy the cocycle property, it holds that

$$
T_{i-1}\left(\theta_{T_{j-i}(\omega)} \omega\right)-T_{m}\left(\theta_{T_{j-i}(\omega)} \omega\right)=T_{i-1-m}\left(\theta_{T_{j+m-i}(\omega)} \omega\right)=-T_{-i+m+1}\left(\theta_{T_{j-1}(\omega)} \omega\right)
$$

and thus

$$
\begin{aligned}
\left|\Phi\left(i, j-i, \omega, u_{0}\right)\right| \leq & \left(1+k_{1}\right)^{i-1} e^{\frac{\lambda_{1}}{2} T_{-i+1}\left(\theta_{T_{j-1}}(\omega) \omega\right)}\left(k_{0} \rho(j-i, \omega)+k_{2}\right) \\
& +\sum_{m=1}^{i-1} 2 k_{2}\left(1+k_{1}\right)^{i-1-m} e^{\frac{\lambda_{1}}{2} T_{-i+m+1}\left(\theta_{T_{j-1}}(\omega) \omega\right)} \\
= & \left(1+k_{1}\right)^{i-1} e^{\frac{\lambda_{1}}{2} T_{-i+1}\left(\theta_{T_{j-1}}(\omega) \omega\right)}\left(k_{0} \rho(j-i, \omega)+k_{2}\right) \\
& +\sum_{m=2-i}^{0} 2 k_{2}\left(1+k_{1}\right)^{-m} e^{\frac{\lambda_{1}}{2} T_{m}\left(\theta_{T_{j-1}(\omega)} \omega\right)} .
\end{aligned}
$$

Note that for every $\epsilon>0$ there is an $m_{\epsilon}>0$ such that for $m<0,|m|>m_{\epsilon}$, then $\left|T_{m}\left(\theta_{T_{j-1}(\omega)} \omega\right)\right|>(d-\epsilon)|m|$, or equivalently, $\frac{\lambda_{1}}{2} T_{m}\left(\theta_{T_{j-1}(\omega)} \omega\right)<m \frac{\lambda_{1}}{2}(d-\epsilon)$, which is a consequence of the second inequality in (3.18). Therefore,

$$
\begin{aligned}
\left(1+k_{1}\right)^{-m} e^{\frac{\lambda_{1}}{2} T_{m}\left(\theta_{T_{j-1}(\omega)} \omega\right)} & =e^{-m \log \left(1+k_{1}\right)+\frac{\lambda_{1}}{2} T_{m}\left(\theta_{T_{j-1}(\omega)} \omega\right)} \\
& \leq e^{m\left(-\log \left(1+k_{1}\right)+\frac{\lambda_{1}}{2}(d-\epsilon)\right)} \leq e^{\nu m}
\end{aligned}
$$

where the last estimate is true since $\frac{\lambda_{1}}{2} d-\log \left(1+k_{1}\right)-\nu-\frac{\lambda_{1}}{2} \epsilon>0$ for small $\epsilon>0$, which follows from the last inequality of (3.18). Hence, the sum on the right-hand side of (3.24) converges to $\frac{R(j)}{2}$ when $i \rightarrow \infty$.

On the other hand, the first term in (3.24) converges to zero for $i \rightarrow \infty$ due to (3.18).

To obtain the absorbing property it remains to mention that, thanks to the cocycle property for $\Phi$, for sufficient large $i \in Z^{+}$,

$$
\begin{aligned}
\left|\Phi\left(i+1, j-i-1, \omega, v_{0}\right)\right|= & \left|\Phi\left(i, j-i, \omega, \Phi\left(1, j-i-1, \omega, v_{0}\right)\right)\right| \\
\leq & \left(1+k_{1}\right)^{i} e^{\frac{-\lambda_{1}}{2} T_{i}\left(\theta_{T_{j-i-1}(\omega)} \omega\right)} \\
& \times\left(k_{0}\left|\Phi\left(1, j-i-1, \omega, v_{0}\right)\right|_{V_{\beta}}+k_{2}\right)+R(j) / 2,
\end{aligned}
$$

Copyright (c) by SIAM. Unauthorized reproduction of this article is prohibited. 
hence

$$
\sup _{v_{0} \in D(j-i-1)}\left|\Phi\left(i+1, j-i-1, \omega, v_{0}\right)\right| \leq \sup _{u_{0} \in F(j-i, \omega)}\left|\Phi\left(i, j-i, \omega, u_{0}\right)\right|<R(j)
$$

for sufficient large $i \in Z^{+}$.

LEMma 3.10. Under the conditions of Lemma 3.9, the absorbing set $B(\omega)$ given by (3.21) is contained in $\mathcal{D}_{Z, V}^{\nu}$.

Proof. We show that $(R(i))_{i \in Z}$ is $\nu$-exponentially growing, for which we will use (3.18). Since

$$
T_{m}\left(\theta_{T_{i-1}(\omega)} \omega\right)=T_{m+i-1}(\omega)-T_{i-1}(\omega)=T_{m+i-1}(\omega)+T_{-i+1}\left(\theta_{T_{i-1}(\omega)} \omega\right),
$$

we obtain that

$$
\begin{aligned}
R(i) & =2 \sum_{m=-\infty}^{0} 2 k_{2}\left(1+k_{1}\right)^{-m} e^{\frac{\lambda_{1}}{2} T_{m}\left(\theta_{T_{i-1}(\omega)} \omega\right)} \\
& =2 \sum_{m=-\infty}^{0} 2 k_{2}\left(1+k_{1}\right)^{-m} e^{\frac{\lambda_{1}}{2}\left(T_{m+i-1}(\omega)+T_{-i+1}\left(\theta_{T_{i-1}}(\omega) \omega\right)\right)} .
\end{aligned}
$$

Furthermore, due to (3.18), for any sufficiently small $\delta$ there exists $j_{0}(\delta) \in Z^{-}$such that for $j \leq j_{0}(\delta)$ we have

$$
T_{j}(\omega) \leq d j+\frac{\delta}{2}|j|
$$

which together with $T_{-i+1}\left(\theta_{T_{i-1}(\omega)} \omega\right) \leq-i+1$ implies that

$$
\begin{aligned}
R(i) & \leq 2 \sum_{m=-\infty}^{0} 2 k_{2}\left(1+k_{1}\right)^{-m} e^{\frac{\lambda_{1}}{2}\left(\left(T_{m+i-1}(\omega)-d(i+m-1)\right)+d(i+m-1)-(i-1)\right)} \\
& \leq 2 \sum_{m=-\infty}^{0} 2 k_{2}\left(1+k_{1}\right)^{-m} e^{\frac{\lambda_{1}}{2}(\delta|i+m-1|+d(i+m-1)-(i-1))} \\
& \leq 2 e^{\frac{\lambda_{1}}{2} \delta|i-1|} e^{(1-d)|i-1|} \sum_{m=-\infty}^{0} 2 k_{2}\left(1+k_{1}\right)^{-m} e^{\frac{\lambda_{1}}{2}(\delta|m|+d m)} .
\end{aligned}
$$

For sufficiently small $\delta$ the sum on the right-hand side is finite. Moreover, for $\nu>$ $(1-d)($ see $(3.19))$ and $\delta>0$ small

$$
\lim _{i \rightarrow-\infty} e^{\nu i} R(i) \leq 2 e^{\frac{\lambda_{1}}{2} \delta} \lim _{i \rightarrow-\infty} e^{\frac{\lambda_{1}}{2} \delta|i|+\nu i} e^{(1-d)|i-1|} \sum_{m=-\infty}^{0} 2 k_{2}\left(1+k_{1}\right)^{-m} e^{\frac{\lambda_{1}}{2}(\delta|m|+d m)}=0
$$

such that $B \subset \mathcal{D}_{Z, V}^{\nu}$.

The conditions of the last two lemmas are always grantable if the nonautonomous perturbation is small in the following sense: $\mu$ could be chosen small. Then, if $\nu>0$ is also small, $d$ should satisfy essentially the next three conditions:

$$
d<1, \quad d+\nu>1, \quad d>2 \nu / \lambda_{1} .
$$

And we can always find an appropriate $d$ solving these three inequalities. Note that the worst case happens when the first eigenvalue $\lambda_{1}$ is small, which forces $\nu$ to be chosen small enough and $d$ to be close to one. But $d$ close to one means that the stopping times $T_{i}$ are close to one in the average, in the sense of the second inequality in (3.18), or in other words, that the contribution of $\||\omega|\|_{\beta^{\prime}, 0, \tau}$ for the construction of the stopping time is small in the average; see (3.9).

Copyright $@$ by SIAM. Unauthorized reproduction of this article is prohibited. 
TheOREM 3.11. Consider $\omega \in \Omega$ such that the assumptions of Lemma 3.9 hold. Then the discrete nonautonomous dynamical system $\Phi(\cdot, \omega)$ has a pullback attractor $\{\mathcal{A}(i, \omega)\}_{i \in Z}$ with respect to the system of $\nu$-exponentially growing sets $\mathcal{D}_{Z, V}^{\nu}$.

Proof. Note that $\Phi(i, j, \omega, \cdot)$ is continuous on $V$. Let $B(\omega) \subset \mathcal{D}_{Z, V}^{\nu}$ be the absorbing set from Lemma 3.9. For any $j \in Z$, let $\tilde{T}(B, j, \omega)$ be the absorption time of $B(j, \omega) \in \mathcal{D}_{Z, V}^{\nu}$ by itself. Set $T^{*}=T^{*}(j)=\tilde{T}(B, j, \omega)$. Notice that $\Phi\left(T^{*},-T^{*}-1+j, \omega, B\left(-T^{*}-1+j, \omega\right)\right)$ is absorbing, and set

$$
C(j, \omega):=\overline{\Phi\left(1,-1+j, \omega, \Phi\left(T^{*},-T^{*}-1+j, \omega, B\left(-T^{*}-1+j, \omega\right)\right)\right.} \subset B(j, \omega) .
$$

Hence, the definition of $T^{*}$ ensures that $C(\omega)=(C(j, \omega))_{j \in Z}$ is $\mathcal{D}_{Z, V}^{\nu}$-pullback absorbing and contained in $\mathcal{D}_{Z, V}^{\nu}$. In addition, by (3.7) and the compact embedding of $V_{\beta} \subset V$ the sets $C(j, \omega)$ are also compact. Now we can apply Theorem 2.2, giving the existence of a pullback attractor $\{\mathcal{A}(i, \omega)\}_{i \in Z}$ for $\Phi$.

The conclusion of the last theorem will be used to study $\varphi$ as a nonautonomous dynamical system. In particular we show that this dynamical system has a pullback attractor.

3.3. Attracting sets for the nonautonomous dynamical systems associated to (3.4). We now study the nonautonomous dynamical system $\varphi$ given by (3.4). For this purpose it would be enough to consider this mapping $\varphi$ along one orbit $\bigcup_{t \in R}\left\{\theta_{t} \omega\right\}$ for a fixed arbitrary $\omega \in \Omega$. However, we are going to consider measurable mappings $\varphi$ on the entire set $\Omega$. In this sense the following definition is given.

Consider the family of closed nonempty sets $(D(\omega))_{\omega \in \Omega} \subset \mathcal{D}_{R, V}^{0}$ with $D(\omega)=$ $B_{V}(0, r(\omega))$ such that

$$
\limsup _{R \ni t \rightarrow-\infty} \frac{\log ^{+} r\left(\theta_{t} \omega\right)}{|t|}=0 .
$$

For such a $D$ define the sets $(G(i, \omega))_{i \in Z}$ by

$$
G(i, \omega)=\overline{\bigcup_{\tau \in\left(T_{i-1}(\omega), T_{i}(\omega)\right]} D\left(\theta_{\tau} \omega\right),}, \quad i \in Z,
$$

which are elements of $\mathcal{D}_{Z, V}^{0}$. In the contrary case, we would find an $\omega$, a subsequence $\left(i^{\prime}(i, \omega)\right)_{i \in Z^{-}}, i^{\prime}(i, \omega):=i^{\prime} \in Z^{-}, t_{i^{\prime}} \in\left[T_{i^{\prime}-1}(\omega), T_{i^{\prime}}(\omega)\right]$, and $u_{i^{\prime}} \in D\left(\theta_{t_{i^{\prime}}} \omega\right)$ such that

$$
0<\limsup _{i^{\prime} \rightarrow-\infty} \frac{\log ^{+}\left|u_{i^{\prime}}\right|}{\left|i^{\prime}\right|} .
$$

But we can estimate this expression by

$$
\limsup _{i^{\prime} \rightarrow-\infty} \frac{\log ^{+}\left|u_{i^{\prime}}\right|}{\left|T_{i^{\prime}-1}(\omega)\right|} \limsup _{i^{\prime} \rightarrow-\infty} \frac{\left|T_{i^{\prime}-1}(\omega)\right|}{\left|i^{\prime}\right|} \leq \limsup _{i^{\prime} \rightarrow-\infty} \frac{\log ^{+}\left|u_{i^{\prime}}\right|}{\left|t_{i^{\prime}}\right|} \limsup _{i^{\prime} \rightarrow-\infty} \frac{\left|T_{i^{\prime}-1}(\omega)\right|}{\left|i^{\prime}\right|}=0,
$$

due to the fact that $u_{i^{\prime}} \in D\left(\theta_{t_{i^{\prime}}} \omega\right)$. Let us emphasize that, by the definition of the stopping times, the last factor in the last term is finite.

We then can conclude the following.

Lemma 3.12. For $D \in \mathcal{D}_{R, V}^{0}$ we have that

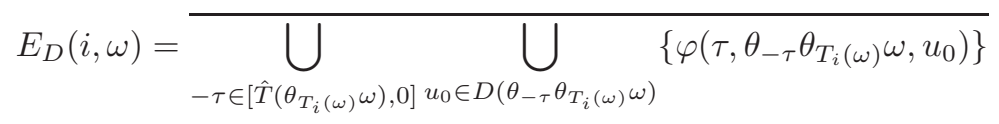

Copyright (C) by SIAM. Unauthorized reproduction of this article is prohibited. 
defines a set in $\mathcal{D}_{Z, V}^{0}$. In addition,

$$
H_{D}(i, \omega)=\overline{\bigcup_{\tau \in\left[0, T\left(\theta_{T_{i}(\omega)} \omega\right)\right]} \bigcup_{u_{0} \in D(i, \omega)}\left\{\varphi\left(\tau, \theta_{T_{i}(\omega)} \omega, u_{0}\right)\right\}}
$$

is in $\mathcal{D}_{Z, V}^{\nu}$ provided $D \in \mathcal{D}_{Z, V}^{\nu}$.

Proof. First, similar to the proof of Lemma 3.9 we obtain that

$$
(1-c \mu)\|u\|_{\beta, \sim, 0, T\left(\theta_{T_{j-1}(\omega)} \omega\right)} \leq c\left(\left|u_{0}\right|+\mu\right) .
$$

On the other hand, for $-s \in\left[\hat{T}\left(\theta_{T_{i}(\omega)} \omega\right), 0\right]$, due to (3.5) we have

$$
\begin{aligned}
\left|\varphi\left(s, \theta_{-s} \theta_{T_{i}(\omega)} \omega, u_{0}\right)\right| & \leq c\left|u_{0}\right|+\left|\int_{0}^{s} S(s-r) G(u(r)) d \theta_{-s} \theta_{T_{i}(\omega)} \omega\right| \\
& \leq c\left|u_{0}\right|+c\left(1+\|u\|_{\beta, \sim, 0, T\left(\theta_{T_{i}(\omega)} \omega\right)}\right)\left\||| \theta_{-s} \theta_{T_{i}(\omega)} \omega \mid\right\|_{\beta^{\prime}, 0, s},
\end{aligned}
$$

and because $\left\||| \theta_{-s} \theta_{T_{i}(\omega)} \omega\left|\left\|_{\beta^{\prime}, 0, s} \leq\right\|\left\|\theta_{T_{i}(\omega)} \omega \mid\right\|_{\beta^{\prime}, \hat{T}\left(\theta_{T_{i}(\omega)} \omega\right), 0} \leq \mu\right.\right.$, we obtain that

$$
\left|\varphi\left(s, \theta_{-s} \theta_{T_{i}(\omega)} \omega, u_{0}\right)\right| \leq c\left|u_{0}\right|+c \mu\left(1+\frac{c}{1-c \mu}\left|u_{0}\right|+\frac{c \mu}{1-c \mu}\right) .
$$

Hence the norm of any element in $E(i, \omega)$ is bounded by

$$
\sup _{u_{0} \in G(i, \omega)} c\left|u_{0}\right|\left(1+\frac{c \mu}{1-c \mu}\right)+c \mu\left(1+\frac{c \mu}{1-c \mu}\right)
$$

for $G(i, \omega)$ introduced in (3.25), which gives the desired property.

The second property follows similarly.

Lemma 3.13. Under the conditions of Theorem 3.11, define the family of sets $\mathcal{A}$ by $\mathcal{A}(\omega):=\mathcal{A}(0, \omega)$, where $\mathcal{A}(i, \omega)$ has been defined in Theorem 3.11. Then $\mathcal{A}$ is invariant and attracting for elements from $\mathcal{D}_{R, V}^{0}$ with respect to $\varphi$.

Proof. For $t>0$ there exists a unique $i^{*}=i^{*}(\omega) \in Z^{-}$such that $-t \in$ $\left(T_{i^{*}(\omega)-1}(\omega), T_{i^{*}(\omega)}(\omega)\right]$. Because of $T_{-i^{*}}\left(\theta_{T_{i^{*}}} \omega\right)=-T_{i^{*}}(\omega)$, due also to the cocycle property and the relationship between $\varphi$ and $\Phi$, we can conclude that for $D \in \mathcal{D}_{R, V}^{0}$

$$
\begin{aligned}
\varphi( & \left.t, \theta_{-t} \omega, D\left(\theta_{-t} \omega\right)\right) \\
& =\varphi\left(T_{-i^{*}}\left(\theta_{T_{i^{*}}} \omega\right), \theta_{T_{i^{*}}(\omega)} \omega, \varphi\left(t-T_{-i^{*}}\left(\theta_{T_{i^{*}}} \omega\right), \theta_{-t-T_{i^{*}}} \theta_{T_{i^{*}}} \omega, D\left(\theta_{-t-T_{i^{*}}} \theta_{T_{i^{*}}} \omega\right)\right)\right) \\
& =\Phi\left(-i^{*}, i^{*}, \omega, \varphi\left(t-T_{-i^{*}}\left(\theta_{T_{i^{*}}} \omega\right), \theta_{-t-T_{i^{*}}} \theta_{T_{i^{*}}} \omega, D\left(\theta_{-t-T_{i^{*}}} \theta_{T_{i^{*}}} \omega\right)\right)\right) \\
& \subset \Phi\left(-i^{*}, i^{*}, \omega, E_{D}\left(i^{*}, \omega\right)\right),
\end{aligned}
$$

where $E_{D} \in \mathcal{D}_{Z, V}^{0}$ is defined by (3.26) and which can be also written as

$$
E_{D}(i, \omega)=\overline{\bigcup_{\tau \in\left[T_{i-1}(\omega), T_{i}(\omega)\right]} \bigcup_{u_{0} \in D\left(\theta_{\tau} \omega\right)}\left\{\varphi\left(T_{i}(\omega)-\tau, \theta_{\tau-T_{i}(\omega)} \theta_{T_{i}(\omega)} \omega, u_{0}\right)\right\}} .
$$

Hence $\mathcal{A}$ attracts $D$ with respect to $\varphi$ since

$$
0 \leq \operatorname{dist}\left(\varphi\left(t, \theta_{-t} \omega, D\left(\theta_{-t} \omega\right)\right), \mathcal{A}(\omega)\right) \leq \operatorname{dist}\left(\Phi\left(-i, i, \omega, E_{D}(i, \omega)\right), \mathcal{A}(0, \omega)\right),
$$

and

$$
\lim _{i \rightarrow-\infty} \operatorname{dist}\left(\Phi\left(-i, i, \omega, E_{D}(i, \omega)\right), \mathcal{A}(0, \omega)\right)=0
$$

Copyright $@$ by SIAM. Unauthorized reproduction of this article is prohibited. 
Now we prove the invariance of $\mathcal{A}$. For $t>0$ we denote by $i^{\prime}(\omega)$ the largest $i$ such that $T_{i^{\prime}}(\omega)<t$ for any $\omega \in \Omega$. Hence by (3.11) for $t_{2}=t, t_{1}=T_{i^{\prime}}(\omega)$

$$
\begin{aligned}
\cdots & \leq T_{i^{\prime}-1}(\omega)<T_{-1}\left(\theta_{t} \omega\right)+t \leq T_{i^{\prime}}(\omega)<t \\
& \leq T_{i^{\prime}+1}(\omega)<T_{1}\left(\theta_{t} \omega\right)+t \leq T_{i^{\prime}+2}(\omega)<T_{2}\left(\theta_{t} \omega\right)+t \leq \cdots .
\end{aligned}
$$

Since $\left(\mathcal{A}\left(i^{\prime}+j, \omega\right)\right)_{j \in Z}$ is pullback attracting with respect to $\Phi$, by (3.23) the same property is true for the following compact set:

$$
\tilde{\mathcal{A}}\left(j, \theta_{t} \omega\right):=\varphi\left(t-T_{i^{\prime}+j}(\omega)+T_{j}\left(\theta_{t} \omega\right), \theta_{T_{i^{\prime}+j}(\omega)} \omega, \mathcal{A}\left(i^{\prime}+j, \omega\right)\right) .
$$

Because of $\left|t-T_{i^{\prime}+j}(\omega)+T_{j}\left(\theta_{t} \omega\right)\right| \leq 1$ and $\{\mathcal{A}(j, \omega)\}_{j \in Z} \in \mathcal{D}_{Z, V}^{\nu}$, applying the second part of Lemma 3.12 we have that $\tilde{\mathcal{A}}\left(j, \theta_{t} \omega\right) \in \mathcal{D}_{Z, V}^{\nu}$. Furthermore

$$
\begin{aligned}
& \Phi\left(1, j, \theta_{t} \omega, \tilde{\mathcal{A}}\left(j, \theta_{t} \omega\right)\right) \\
& \quad=\varphi\left(T\left(\theta_{T_{j}\left(\theta_{t} \omega\right)} \theta_{t} \omega\right), \theta_{T_{j}\left(\theta_{t} \omega\right)} \theta_{t} \omega, \varphi\left(t+T_{j}\left(\theta_{t} \omega\right)-T_{i^{\prime}+j}(\omega), \theta_{T_{i^{\prime}+j}(\omega)} \omega, \mathcal{A}\left(i^{\prime}+j, \omega\right)\right)\right) \\
& \quad=\varphi\left(t+T_{j+1}\left(\theta_{t} \omega\right)-T_{i^{\prime}+j}(\omega), \theta_{T_{i^{\prime}+j}(\omega)} \omega, \mathcal{A}\left(i^{\prime}+j, \omega\right)\right) \\
& \quad=\varphi\left(t+T_{j+1}\left(\theta_{t} \omega\right)-T_{i^{\prime}+j+1}(\omega), \theta_{T_{i^{\prime}+j+1}(\omega)} \omega, \mathcal{A}\left(i^{\prime}+j+1, \omega\right)\right)=\tilde{\mathcal{A}}\left(j+1, \theta_{t} \omega\right),
\end{aligned}
$$

which shows that $\tilde{\mathcal{A}}\left(\cdot, \theta_{t} \omega\right)$ is a compact invariant set in $\mathcal{D}_{Z, V}^{\nu}$ for the cocycle $\Phi\left(\cdot, \cdot, \theta_{t} \omega, \cdot\right)$. Since $\tilde{\mathcal{A}}\left(\cdot, \theta_{t} \omega\right) \in \mathcal{D}_{Z, V}^{\nu}$, it is attracted by $\mathcal{A}\left(\cdot, \theta_{t} \omega\right)$, and due to the invariance of the former

$$
\tilde{\mathcal{A}}\left(j, \theta_{t} \omega\right) \subset \mathcal{A}\left(j, \theta_{t} \omega\right)
$$

Now we define

$$
\hat{\mathcal{A}}\left(i^{\prime}+j+1, \omega\right):=\varphi\left(T_{i^{\prime}+j+1}(\omega)-t-T_{j}\left(\theta_{t} \omega\right), \theta_{T_{j}\left(\theta_{t} \omega\right)} \theta_{t} \omega, \mathcal{A}\left(j, \theta_{t} \omega\right)\right),
$$

which implies that $\hat{\mathcal{A}}$ has qualitatively the same properties as $\tilde{\mathcal{A}}$. In particular, $\hat{\mathcal{A}}(j, \omega) \subset \mathcal{A}(j, \omega)$. But we have

$$
\begin{aligned}
\mathcal{A}\left(\theta_{t} \omega\right) & =\mathcal{A}\left(0, \theta_{t} \omega\right) \\
& =\Phi\left(1,-1, \theta_{t} \omega, \mathcal{A}\left(-1, \theta_{t} \omega\right)\right)=\varphi\left(t-T_{i^{\prime}}(\omega), \theta_{T_{i^{\prime}}}(\omega) \omega, \hat{\mathcal{A}}\left(i^{\prime}(\omega), \omega\right)\right) \\
& \subset \varphi\left(t-T_{i^{\prime}}(\omega), \theta_{T_{i^{\prime}}(\omega)} \omega, \mathcal{A}\left(i^{\prime}(\omega), \omega\right)\right)=\tilde{\mathcal{A}}\left(0, \theta_{t} \omega\right)
\end{aligned}
$$

such that, by (3.28) and (3.29), $\tilde{\mathcal{A}}\left(0, \theta_{t} \omega\right)=\mathcal{A}\left(\theta_{t} \omega\right)$ and

$$
\varphi(t, \omega, \mathcal{A}(\omega))=\varphi\left(t-T_{i^{\prime}}(\omega), \theta_{T_{i^{\prime}}(\omega)} \omega, \Phi\left(i^{\prime}, 0, \omega, \mathcal{A}(0, \omega)\right)=\mathcal{A}\left(\theta_{t} \omega\right),\right.
$$

and hence $\mathcal{A}$ is invariant.

Lemma 3.14. Suppose that

$$
1 \geq \hat{d}:=\limsup _{i \rightarrow-\infty} \frac{\left|T_{i}(\omega)\right|}{|i|}, \quad \check{d}:=\liminf _{i \rightarrow-\infty} \frac{\left|T_{i}(\omega)\right|}{|i|} \geq d>0 .
$$

(i) We have that $\mathcal{A} \in \mathcal{D}_{R, V}^{\nu / \check{d}}$.

(ii) Suppose that $\hat{d}>\check{d}$ and $\nu>0$. Then there exists an $\omega \in \Omega$ and $D \in \mathcal{D}_{R, V}^{\nu / \check{d}}$ such that the restriction of $t \rightarrow D\left(\theta_{t} \omega\right)$ to $\left(T_{i}(\omega)\right)_{i \in Z}$ is in $\mathcal{D}_{Z, V}^{\nu \hat{d} / \breve{d}}$ but not in $\mathcal{D}_{Z, V}^{\nu}$ along an orbit $\bigcup_{t \in R}\left\{\theta_{t} \omega\right\}$. 
(iii) If $D \in \mathcal{D}_{R, V}^{0}$ then the restriction of $t \rightarrow D\left(\theta_{t} \omega\right)$ to $\left(T_{i}(\omega)\right)_{i \in N}$ is in $\mathcal{D}_{Z, V}^{0}$.

Proof. (i) As $\mathcal{A}\left(\theta_{T_{i}(\omega)} \omega\right)=\mathcal{A}(i, \omega)$, we obtain

$$
\limsup _{i \rightarrow-\infty} \frac{\log ^{+}\left(\sup _{u \in \mathcal{A}\left(\theta_{T_{i}(\omega)} \omega\right)}|u|\right)}{\left|T_{i}(\omega)\right|} \leq \limsup _{i \rightarrow-\infty} \frac{\log ^{+}\left(\sup _{u \in \mathcal{A}(i, \omega)}|u|\right)}{|i|} \limsup _{i \rightarrow-\infty} \frac{|i|}{\left|T_{i}(\omega)\right|} \leq \frac{\nu}{\tilde{d}} .
$$

In addition, we can show a similar estimate for $\left|\varphi\left(t, \theta_{T_{i}(\omega)} \omega, u_{0}\right)\right|$ as in (3.27). By using that estimate we obtain the desired result simply taking $t \in\left(T_{i-1}(\omega), T_{i}(\omega)\right]$ and applying the above convergence result.

(ii) Let $i^{\prime}$ be a sequence such that

$$
\limsup _{i \rightarrow-\infty} \frac{\left|T_{i}(\omega)\right|}{|i|}=\lim _{i^{\prime} \rightarrow-\infty} \frac{\left|T_{i^{\prime}}(\omega)\right|}{\left|i^{\prime}\right|} .
$$

On some orbit $\bigcup_{t \in R}\left\{\theta_{t} \omega\right\}$ we define an element $D \in \mathcal{D}_{R, V}^{\nu / \check{d}}$ by $D\left(\theta_{t} \omega\right)=\{0\}$ for $t \neq T_{i^{\prime}}(\omega)$. In addition, for a sufficiently small $\epsilon>0$ we choose a sequence $\left(u_{i^{\prime}}\right)_{i^{\prime}}$ such that

$$
\lim _{i^{\prime} \rightarrow-\infty} \frac{\log ^{+}\left|u_{i^{\prime}}\right|}{\left|T_{i^{\prime}(\omega)}\right|}=\frac{\nu}{\check{d}}-\epsilon
$$

and set $D\left(\theta_{T_{i^{\prime}}(\omega)} \omega\right)=\left\{u_{i^{\prime}}\right\}$. We have that

$$
\begin{aligned}
\limsup _{i \rightarrow-\infty} \frac{\log ^{+}\left(\sup _{u \in D\left(\theta_{T_{i}(\omega)} \omega\right)}|u|\right)}{|i|} & =\limsup _{i \rightarrow-\infty}\left(\frac{\log ^{+}\left(\sup _{u \in D\left(\theta_{T_{i}(\omega)} \omega\right)}|u|\right)}{\left|T_{i}(\omega)\right|} \frac{\left|T_{i}(\omega)\right|}{|i|}\right) \\
& =\lim _{i^{\prime} \rightarrow-\infty}\left(\frac{\log ^{+}\left(\sup _{u \in D\left(\theta_{T_{i^{\prime}}(\omega)} \omega\right)}|u|\right)}{\left|T_{i^{\prime}}(\omega)\right|} \frac{\left|T_{i^{\prime}}(\omega)\right|}{\left|i^{\prime}\right|}\right) \\
& =\left(\frac{\nu}{\check{d}}-\epsilon\right) \hat{d}<\frac{\nu \hat{d}}{\check{d}}
\end{aligned}
$$

and it is bigger than $\nu$ when $\epsilon>0$ is chosen sufficiently small.

(iii) This follows similar to the proof of (ii).

We would like to emphasize the following: by part (ii) in the above result we know that the restriction of particular elements $D \in \mathcal{D}_{R, V}^{\nu / \check{d}}$ to the discrete time sequence $\left(T_{i}(\omega)\right)_{i \in Z}$ is only in $\mathcal{D}_{Z, V}^{\hat{d} \nu / \breve{d}}$ but not in $\mathcal{D}_{Z, V}^{\nu}$ in general. Therefore, that restriction is not attracted by $(\mathcal{A}(i, \omega))_{i \in Z}$ if $\hat{d}>\check{d}$, and consequently the set $\mathcal{A}$ is not an attractor for $\varphi$, since in that case it should attract all sets in $\mathcal{D}_{R, V}^{\nu / \breve{d}}$.

By these reasons $\mathcal{A}$ is only called a partially attracting set, since it is contained in $\mathcal{D}_{R, V}^{\nu / \breve{d}}$ but we cannot prove that $\mathcal{A}$ is attracting for the entire system $\mathcal{D}_{R, V}^{\nu / \breve{d}}$. However, in Theorem 4.6 below and in a measurable setting, we will prove stronger growth conditions for $\mathcal{A}$, which will allow us to conclude that $\mathcal{A}$ is a (random) attractor.

4. Attractors for random dynamical systems of SPDEs with fractional Brownian motion. In this section we study the nonautonomous dynamical system under measurability assumptions. In particular, we need to introduce a metric dynamical system. It will be crucial that the integrals with Hölder continuous integrators are defined pathwise. This is a qualitative difference to the definition of the classical stochastic integral where the integrand is a white noise. We recall that the pathwise 
definition of the former integral just gave us the nonautonomous dynamical system $\varphi$ from section 3.1 defined by (3.8).

A one-dimensional $\mathrm{fBm}$ is a centered Gauss process on $R$ with Hurst parameter $H \in(0,1)$ having the covariance

$$
R(s, t)=\frac{1}{2}\left(|t|^{2 H}+|s|^{2 H}-|t-s|^{2 H}\right)
$$

defined on an appropriate probability space. Similarly we can define an $\mathrm{fBm}$ with values in $V$ and covariance $Q$, where $Q$ is a positive symmetric operator on $V$ of trace class. It is known that $Q$ has a discrete spectrum $\left(q_{i}\right)_{i \in N}$ related to the complete orthonormal system in $V$ given by $\left(f_{i}\right)_{i \in N}$. This process has a version $\omega$ in $C_{0}(R, V)$, the space of continuous functions which are zero at zero, and $q_{i}^{-\frac{1}{2}} \pi_{i} \omega$ are independently and identically distributed one-dimensional fBm, where $\pi_{i}$ is the projection on the $i$ th mode of the base $\left(f_{i}\right)_{i \in N}$, such that we can define the integrals as in subsection 2.2. For simplicity we identify in the following the base $\left(f_{i}\right)_{i \in N}$ with the base $\left(e_{i}\right)_{i \in N}$.

In what follows we consider a canonical version of this process given by the probability space $\left(C_{0}(R, V), \mathcal{B}\left(C_{0}(R, V)\right), P\right)$, where $P$ is the Gauss measure generated by the fBm. On this probability space we can also introduce the shift $\theta_{t} \omega(\cdot)=$ $\omega(\cdot+t)-\omega(t)$.

Lemma 4.1. $\left(C_{0}(R, V), \mathcal{B}\left(C_{0}(R, V)\right), P, \theta\right)$ is an ergodic metric dynamical system.

The proof of this lemma can be found in [21] and with a deeper analysis in [14].

This (canonical) process has a version which is $\beta^{\prime \prime}$-Hölder continuous on any interval $[-k, k]$ for $\beta^{\prime \prime}<H$.

Let us denote by $\Omega_{\beta^{\prime \prime}} \subset C_{0}(R, V)$ the set of elements which are $\beta^{\prime \prime}$-Hölder continuous on any interval $[-k, k], k \in N$, and are zero at 0 .

Lemma 4.2 (see [5]). We have $\Omega_{\beta^{\prime \prime}} \in \mathcal{B}\left(C_{0}(R, V)\right)$. In addition, $\Omega_{\beta^{\prime \prime}}$ is $\left(\theta_{t}\right)_{t \in R^{-}}$ invariant.

Choose $\mu, \nu$ such that (3.18) holds. Then by the ergodic theory there exists a $\left(\theta_{t}\right)_{t \in R}$-invariant set $\Omega_{1} \in \mathcal{B}\left(C_{0}(R, V)\right)$ of full measure such that

$$
\begin{aligned}
& \lim _{t \rightarrow \pm \infty} \frac{1}{t} \int_{0}^{t}\left(\frac{\sup _{r \in[0,1]}||\left|\theta_{r+q} \omega\right| \|_{\beta^{\prime \prime},-1,0}+\mu}{\mu}\right)^{\frac{1}{\beta^{\prime \prime}-\beta^{\prime}}} d q \\
& \quad=E_{P}\left(\frac{\sup _{r \in[0,1]}||\left|\theta_{r} \omega\right| \|_{\beta^{\prime \prime},-1,0}+\mu}{\mu}\right)^{\frac{1}{\beta^{\prime \prime}-\beta^{\prime}}}=: d^{-1}<\infty .
\end{aligned}
$$

For the construction of the invariant set $\Omega_{1}$ we refer to Arnold [1, p. 538, Ergodic Theorem (iv)]. The above expectation can be controlled by choosing a $Q$ with small trace such that $d$ is close to 1 ; see, for instance, the construction of the random variable $K$ in Kunita [19, Theorem 1.4.1]. Let us also choose $\operatorname{tr} Q$ small enough such that (3.19) holds true, i.e.,

$$
\nu+d>1
$$

In addition, there exists a $\left(\theta_{t}\right)_{t \in R}$-invariant set $\Omega_{2} \in \mathcal{B}\left(C_{0}(R, V)\right)$ of full measure such that $R \ni t \mapsto \sup _{r \in[0,1]}|| \mid \theta_{t+r} \omega \|_{\beta^{\prime \prime},-1,0}$ has a sublinear growth, which follows by Arnold [1, Proposition 4.1.3]. Indeed, $\sup _{r \in[0,1]}||\left|\theta_{r} \omega\left\|_{\beta^{\prime \prime},-1,0} \leq\right\|\right||\omega| \|_{\beta^{\prime \prime},-1,1}$, where the right-hand side of this inequality has finite moments of any order, by Kunita [19, Theorem 1.4.1].

Setting $\Omega=\Omega_{1} \cap \Omega_{2} \cap \Omega_{\beta^{\prime \prime}}$, let $\mathcal{F}$ be the trace- $\sigma$-algebra of $\mathcal{B}\left(C_{0}(R, V)\right)$ with respect to $\Omega$, and let us consider the restriction of the measure $P$ to this $\sigma$-algebra, 
which we denote again by $P$. Since $\Omega$ is $\left(\theta_{t}\right)_{t \in R}$-invariant it is not hard to see that the restriction of $\theta$ to $R \times \Omega$ is a random flow and that $(\Omega, \mathcal{F}, P, \theta)$ is an ergodic metric dynamical system; see [3] for a general proof of these properties.

We are now in a position to derive from the non-autonomous dynamical system $\varphi$ a random dynamical system.

LEMMA 4.3. (i) Let $\varphi$ be the nonautonomous dynamical system given by (3.8). Then this mapping is $\mathcal{B}\left(R^{+}\right) \otimes \mathcal{F} \otimes \mathcal{B}(V), \mathcal{B}(V)$ measurable, and hence $\varphi$ is a random dynamical system.

(ii) The stopping times $T, \hat{T}$ are measurable.

Proof. First note that $(r, \omega) \mapsto \omega(r)$ is measurable such that $(r, \omega) \mapsto D_{T-}^{1-\alpha} \omega_{T-}[r]$ is measurable. Assuming in addition that $(r, \omega) \mapsto k(r, \omega) \in L_{2}(V)$ is measurable and that for any $\omega$ the product $\left\|D_{0+}^{\alpha} k(\cdot, \omega)[r]\right\|_{L_{2}(V)}\left|D_{T-}^{1-\alpha} \omega_{T-}[r]\right|$ is integrable, then the stochastic integral

$$
\omega \mapsto \int_{0}^{T} S(T-r) G(u(r, \omega)) d \omega
$$

is measurable, since $k(r, \omega)=G(u(r, \omega))$ has the above properties.

Since the solution $u$ of (3.4) is given by the Banach fixed point theorem, it can be constructed by successive iterations in the space $C^{\beta, \sim}([0, T] ; V)$, starting this procedure with the constant function $u_{0}$. Although these approximations are given on different time intervals $\left[T_{i}(\omega), T_{i+1}(\omega)\right]$ they can be finitely concatenated to one approximation on $[0, T]$ converging to a solution of (3.4) in the separable Banach space $C([0, T] ; V)$ which is measurable.

If we pick initial conditions in a sufficiently small neighborhood of $u_{0}$, the contraction constants for the corresponding mapping in the Banach fixed point theorem can be chosen to be the same, and therefore $u_{0} \mapsto \varphi\left(t, \omega, u_{0}\right)$ is continuous. Then, by Castaing and Valadier [4], the mapping $\varphi$ is measurable on $\mathcal{B}([0, T]) \otimes \mathcal{F} \otimes \mathcal{B}(V), \mathcal{B}(V)$. Hence, the $\mathcal{B}\left(R^{+}\right) \otimes \mathcal{F} \otimes \mathcal{B}(V), \mathcal{B}(V)$ measurability of this expression follows immediately.

(ii) Since $\omega \mapsto|||\omega| \|_{\beta^{\prime}, 0, \tau}$ is measurable, the measurability of $\omega \mapsto T(\omega)$ follows. In the same manner we can argue for $\hat{T}$.

In order to establish the growth of the stopping times we need the following lemma.

Lemma 4.4. Let $N(\omega) \in N$ be the random number of stopping times in $[-1,0]$ defined by (3.9) and (3.10). Then we have for $\omega \in \Omega_{\beta^{\prime \prime}}$

$$
N(\omega) \leq\left(\frac{\|\omega\| \|_{\beta^{\prime \prime},-1,0}+\mu}{\mu}\right)^{\frac{1}{\beta^{\prime \prime}-\beta^{\prime}}} .
$$

Proof. The following estimates follow easily on $\Omega_{\beta^{\prime \prime}}$ :

$$
\begin{aligned}
\mu & =\sup _{\hat{T}(\omega) \leq s<t \leq 0} \frac{|\omega(t)-\omega(s)|}{|t-s|^{\beta^{\prime}}}+\mu(-\hat{T}(\omega))^{1-\beta^{\prime}} \\
& \leq\left(\sup _{\hat{T}(\omega) \leq s<t \leq 0} \frac{|\omega(t)-\omega(s)|}{|t-s|^{\beta^{\prime \prime}}}+\mu(-\hat{T}(\omega))^{1-\beta^{\prime \prime}}\right)(-\hat{T}(\omega))^{\beta^{\prime \prime}-\beta^{\prime}} \\
& \leq\left(|| \omega \mid \|_{\beta^{\prime \prime},-1,0}+\mu\right)(-\hat{T}(\omega))^{\beta^{\prime \prime}-\beta^{\prime}}
\end{aligned}
$$

such that

$$
|\hat{T}(\omega)| \geq\left(\frac{\mu}{\||\omega|\|_{\beta^{\prime \prime},-1,0}+\mu}\right)^{\frac{1}{\beta^{\prime \prime}-\beta^{\prime}}}
$$

Copyright (c) by SIAM. Unauthorized reproduction of this article is prohibited. 
The same estimate holds for $\hat{T}\left(\theta_{T_{-i}(\omega)} \omega\right)$ as long as $\hat{T}\left(\theta_{T_{-i}(\omega)} \omega\right)+T_{-i}(\omega)=T_{-i-1}(\omega) \geq$ -1 . For the smallest number $i$ having this property we set $N(\omega)=i+1$. Then due to the definition of the stopping times (3.10) we conclude that

$$
1 \geq\left|T_{-N(\omega)}(\omega)\right|=\sum_{i=0}^{N(\omega)-1}\left|\hat{T}\left(\theta_{T_{-i}(\omega)} \omega\right)\right| \geq N(\omega)\left(\frac{\mu}{\||\omega|\|_{\beta^{\prime \prime},-1,0}+\mu}\right)^{\frac{1}{\beta^{\prime \prime}-\beta^{\prime}}},
$$

and then the result follows.

LEMMA 4.5. On $\Omega$ we have

$$
\liminf _{k \rightarrow-\infty} \frac{\left|T_{k}(\omega)\right|}{|k|}=\liminf _{k \rightarrow-\infty} \frac{T_{k}(\omega)}{k} \geq d>0,
$$

where $d$ has been defined in (4.1). On this set $\left|T\left(\theta_{T_{i}(\omega)} \omega\right)\right|^{-\beta}$ is subexponentially growing for $i \rightarrow-\infty$.

Proof. Let $M_{j}(\omega)$ be the number of stopping times $T_{k}(\omega)$ in $(j-1, j]$.

For $j \in-Z^{+}$let $k_{j}(\omega)$ be the biggest integer such that $T_{k_{j}(\omega)} \leq j$. Then choosing $t_{2}=j$ and $t_{1}=T_{k_{j}(\omega)}(\omega)$ from (3.11) we know

$$
\begin{aligned}
\cdots & <T_{k_{j}(\omega)-2}(\omega) \leq j+\hat{T}\left(\theta_{j} \omega\right)+\hat{T}\left(\theta_{\hat{T}\left(\theta_{j} \omega\right)+j} \omega\right)=j+T_{-2}\left(\theta_{j} \omega\right) \\
& <T_{k_{j}(\omega)-1}(\omega) \leq j+\hat{T}\left(\theta_{j} \omega\right)=j+T_{-1}\left(\theta_{j} \omega\right)<T_{k_{j}(\omega)}(\omega) \leq j
\end{aligned}
$$

such that $M_{j}(\omega)=N\left(\theta_{j} \omega\right)$ or $M_{j}(\omega)=N\left(\theta_{j} \omega\right)-1$, where $N(\omega)$ has been introduced in Lemma 4.4. Hence

$$
\begin{aligned}
\limsup _{n \rightarrow \infty, n>1} \frac{\sum_{j=0}^{n-1} M_{-j}(\omega)}{n-1} & \leq \limsup _{n \rightarrow \infty, n>1} \frac{\sum_{j=0}^{n-1} N\left(\theta_{-j} \omega\right)}{n-1} \\
& \leq \limsup _{n \rightarrow \infty, n>1} \frac{\sum_{j=0}^{n-1}\left(\frac{\left\|\theta_{-j} \omega \mid\right\|_{\beta^{\prime \prime},-1,0}+\mu}{\mu}\right)^{\frac{1}{\beta^{\prime \prime}-\beta^{\prime}}}}{n-1},
\end{aligned}
$$

which follows from Lemma 4.4 for $\omega \in \Omega_{\beta^{\prime \prime}}$. The terms under the sum of the numerator on the right-hand side can be estimated by

$$
\left(\frac{\sup _{r \in[0,1]}||\left|\theta_{r+q-j} \omega\right| \|_{\beta^{\prime \prime},-1,0}+\mu}{\mu}\right)^{\frac{1}{\beta^{\prime \prime}-\beta^{\prime}}} \text { for any } q \in[-1,0]
$$

and hence

$$
N\left(\theta_{-j} \omega\right) \leq \int_{-1}^{0}\left(\frac{\sup _{r \in[0,1]}||\left|\theta_{r+q-j} \omega\right| \|_{\beta^{\prime \prime},-1,0}+\mu}{\mu}\right)^{\frac{1}{\beta^{\prime \prime}-\beta^{\prime}}} d q .
$$

Then we obtain the estimate for $n>1$

$$
\frac{\sum_{j=0}^{n-1} N\left(\theta_{-j} \omega\right)}{n-1} \leq \frac{1}{n-1} \int_{-n}^{0}\left(\frac{\sup _{r \in[0,1]}\left|\left\|\theta_{r+q} \omega \mid\right\|_{\beta^{\prime \prime},-1,0}+\mu\right.}{\mu}\right)^{\frac{1}{\beta^{\prime \prime}-\beta^{\prime}}} d q,
$$

where the right-hand side converges to $1 / d$ for $\omega \in \Omega_{1}$ and $n \rightarrow \infty$ by (4.1). Then we have

$$
\liminf _{n \rightarrow \infty} \frac{\left|T_{-\sum_{j=0}^{n-1} M_{-j}(\omega)}(\omega)\right|}{\sum_{j=0}^{n-1} M_{-j}(\omega)} \geq \liminf _{n \rightarrow \infty} \frac{(n-1)}{\sum_{j=0}^{n-1} M_{j-1}(\omega)} \geq d
$$

Copyright $@$ by SIAM. Unauthorized reproduction of this article is prohibited. 
To see that finally the conclusion holds true, choose for every $k \in-N$ an $n=n(k, \omega)$ such that

$$
-\sum_{j=0}^{n} M_{-j}(\omega)<k \leq-\sum_{j=0}^{n-1} M_{-j}(\omega) .
$$

Thus

$$
\begin{aligned}
\liminf _{k \rightarrow \infty} \frac{\left|T_{-k}(\omega)\right|}{|k|} & \geq \liminf _{n \rightarrow-\infty} \frac{\left|T_{-\sum_{j=0}^{n-1} M_{-j}(\omega)}(\omega)\right|}{\sum_{j=0}^{n} M_{-j}(\omega)} \geq \liminf _{n \rightarrow-\infty} \frac{\left|T_{-\sum_{j=0}^{n-1} M_{-j}(\omega)}(\omega)\right|}{\sum_{j=0}^{n-1} M_{-j}(\omega)+N\left(\theta_{-n} \omega\right)} \\
& =\liminf _{n \rightarrow-\infty} \frac{n-1}{\sum_{j=0}^{n-1} M_{-j}(\omega)} \geq d
\end{aligned}
$$

by the sublinear convergence of $n \mapsto N\left(\theta_{n} \omega\right)$ (thanks to the ergodic theorem) and the at least asymptotical linear growth of $n \mapsto \sum_{j=0}^{n-1} M_{-j}(\omega)+N\left(\theta_{-n} \omega\right)$.

To prove the second part of the statement, note that similar to the estimate of $\hat{T}$ in the proof of Lemma 4.4, for $\omega \in \Omega$,

$$
\left|T\left(\theta_{T_{i}(\omega)} \omega\right)\right|^{-\beta} \leq\left(\frac{\left\|\left|\theta_{T_{i}(\omega)} \omega\right|\right\|_{\beta^{\prime \prime}, 0,1}+\mu}{\mu}\right)^{\frac{\beta}{\beta^{\prime \prime}-\beta^{\prime}}}
$$

for large $|i|$, which follows from the fact that the mapping $i \mapsto||\left|\theta_{T_{i}(\omega)} \omega\right| \|_{\beta^{\prime \prime}, 0,1}$ grows sublinearly in $\Omega \subset \Omega_{2}$, and therefore, the previous inequality shows the subpolynomial growth of $\left|T\left(\theta_{T_{i}(\omega)} \omega\right)\right|^{-\beta}$.

We can finally prove the main result of this section.

THEOREM 4.6. The pullback attractor stated in Lemma 3.13 is a random attractor attracting the random tempered sets $\hat{\mathcal{D}}$, where $\hat{\mathcal{D}}$ has been defined in (2.3).

Proof. Since the stopping times $T_{i}$ are random variables we obtain that the radii of the absorbing balls $R(i, \omega)$ are random variables, and therefore the balls $B(i, \omega)$ are random sets. Note that

$$
\mathcal{A}(\omega)=\bigcap_{j \in Z^{+}} \bigcup_{i \in Z^{+}, i \geq j} \overline{\Phi(i,-i, \omega, B(-i, \omega))} .
$$

$\Phi$ inherits the measurable properties of $\varphi$ and in particular $u \mapsto \Phi(i,-i, \omega, u)$ is continuous. Hence $\overline{\Phi(i,-i, \omega, B(-i, \omega))}$ is a random set. Then by [7] we have that $\mathcal{A}$ is a random set. On the other hand, we have that $\mathcal{A}\left(\theta_{T_{i}(\omega)} \omega\right) \subset B(i, \omega)$ and $\mathcal{A} \in \mathcal{D}_{R, V}^{\frac{\nu}{d}}$ for $\check{d}$ given by (3.30). Furthermore, Lemma 2.1 shows that $\mathcal{A}$ is a set in $\hat{\mathcal{D}}$, and since $\hat{\mathcal{D}} \subset \mathcal{D}_{R, V}^{0}$ the conclusion follows from Lemma 3.13 .

5. Appendix. We present here the proof of Lemma 3.7.

Proof. We have to estimate

$$
\|u\|_{\beta, T_{i-1}\left(\theta_{T_{j}} \omega\right), T_{i}\left(\theta_{T_{j}} \omega\right)}=\sup _{T_{i-1}\left(\theta_{T_{j}} \omega\right) \leq t \leq T_{i}\left(\theta_{T_{j}} \omega\right)}|u(t)|+\|u\|_{\beta, T_{i-1}\left(\theta_{T_{j}} \omega\right), T_{i}\left(\theta_{T_{j}} \omega\right) .} .
$$

First, considering $u_{0} \in V_{\beta}$, from (3.2) and (3.3) we obtain

$$
\left\|S(\cdot) u_{0}\right\|_{\beta, T_{i-1}\left(\theta_{T_{j}} \omega\right), T_{i}\left(\theta_{T_{j}} \omega\right)} \leq c e^{-\lambda_{1} T_{i-1}\left(\theta_{T_{j}} \omega\right)}\left|u_{0}\right|_{V_{\beta}} .
$$

Thanks to the additivity of the above integral (see [5]), for $t \in\left[T_{i-1}\left(\theta_{T_{j}} \omega\right), T_{i}\left(\theta_{T_{j}} \omega\right)\right]$ the following splitting holds true: 


$$
\begin{aligned}
\int_{0}^{t} S(t-r) G(u(r)) d \theta_{T_{j}} \omega= & \int_{T_{i-1}\left(\theta_{T_{j}} \omega\right)}^{t} S(t-r) G(u(r)) d \theta_{T_{j}} \omega \\
& +\sum_{m=1}^{i-1} \int_{T_{m-1}\left(\theta_{T_{j}} \omega\right)}^{T_{m}\left(\theta_{T_{j}} \omega\right)} S(t-r) G(u(r)) d \theta_{T_{j}} \omega,
\end{aligned}
$$

and therefore, in particular,

$$
\begin{aligned}
& \left\|\int_{0} S(\cdot-r) G(u(r)) d \theta_{T_{j}} \omega\right\|_{\beta, T_{i-1}\left(\theta_{T_{j}} \omega\right), T_{i}\left(\theta_{T_{j}} \omega\right)} \\
& \leq\|\| \int_{T_{i-1}\left(\theta_{T_{j}} \omega\right)} S(\cdot-r) G(u(r)) d \theta_{T_{j}} \omega\|\|_{\beta, T_{i-1}\left(\theta_{T_{j}} \omega\right), T_{i}\left(\theta_{T_{j}} \omega\right)} \\
& \quad+\sum_{m=1}^{i-1}\|\| \int_{T_{m-1}\left(\theta_{T_{j}} \omega\right)}^{T_{m}\left(\theta_{T_{j}} \omega\right)} S(\cdot-r) G(u(r)) d \theta_{T_{j}} \omega \|_{\beta, T_{i-1}\left(\theta_{T_{j}} \omega\right), T_{i}\left(\theta_{T_{j}} \omega\right)} .
\end{aligned}
$$

Regarding the first term on the right-hand side of (5.1), notice that for $s<t \in$ $\left[T_{i-1}\left(\theta_{T_{j}} \omega\right), T_{i}\left(\theta_{T_{j}} \omega\right)\right]$, we have that

$$
\begin{aligned}
& \left|\int_{T_{i-1}\left(\theta_{T_{j}} \omega\right)}^{t} S(t-r) G(u(r)) d \theta_{T_{j}} \omega-\int_{T_{i-1}\left(\theta_{T_{j}} \omega\right)}^{s} S(s-r) G(u(r)) d \theta_{T_{j}} \omega\right| \\
& \quad=\left|\int_{s}^{t} S(t-r) G(u(r)) d \theta_{T_{j}} \omega+\int_{T_{i-1}\left(\theta_{T_{j}} \omega\right)}^{s}(S(t-s)-\mathrm{Id}) S(s-r) G(u(r)) d \theta_{T_{j}} \omega\right| \\
& \quad \leq\left|\int_{s^{\prime}}^{t^{\prime}} S\left(t^{\prime}-r\right) G\left(u\left(r+T_{i-1}\left(\theta_{T_{j}} \omega\right)\right)\right) d \theta_{T_{i+j-1}} \omega\right| \\
& \quad+c\left(t^{\prime}-s^{\prime}\right)^{\beta}\left|\int_{0}^{s^{\prime}} S\left(s^{\prime}-r\right) G\left(u\left(r+T_{i-1}\left(\theta_{T_{j}} \omega\right)\right)\right) d \theta_{T_{i+j-1}} \omega\right|_{V_{\beta}},
\end{aligned}
$$

where we have performed the change of variable $\tilde{r}=r-T_{i-1}\left(\theta_{T_{j}} \omega\right)$ and later on renamed $\tilde{r}$ as $r$; see Lemma 2.4. Therefore, in the previous expression, $s^{\prime}=s-$ $T_{i-1}\left(\theta_{T_{j}} \omega\right), t^{\prime}=t-T_{i-1}\left(\theta_{T_{j}} \omega\right)$. Note that then $s^{\prime}<t^{\prime} \in\left[0, T_{1}\left(\theta_{T_{i+j-1}} \omega\right)\right]$.

The first term on the right-hand side of (5.2) can be estimated in the following way:

$$
\begin{aligned}
& \left|\int_{s^{\prime}}^{t^{\prime}} S\left(t^{\prime}-r\right) G\left(u\left(r+T_{i-1}\left(\theta_{T_{j}} \omega\right)\right)\right) d \theta_{T_{i+j-1}} \omega\right| \\
& \quad \leq c\left|\| \theta _ { T _ { i + j - 1 } } \omega | \| _ { \beta ^ { \prime } , 0 , T ( \theta _ { T _ { i + j - 1 } } \omega ) } \int _ { s ^ { \prime } } ^ { t ^ { \prime } } \left(\frac{\left(c_{G}+c_{D G}\left|u\left(r+T_{i-1}\left(\theta_{j} \omega\right)\right)\right|\right) e^{-\lambda_{1}\left(t^{\prime}-r\right)}}{\left(r-s^{\prime}\right)^{\alpha}}\right.\right. \\
& \quad+\int_{s^{\prime}}^{r} \frac{\left(c_{G}+c_{D G}\left|u\left(r+T_{i-1}\left(\theta_{j} \omega\right)\right)\right|\right) e^{-\lambda_{1}\left(t^{\prime}-r\right)}(r-q)^{\beta}}{\left(t^{\prime}-r\right)^{\beta}(r-q)^{\alpha+1}} d q \\
& \left.\quad+\int_{s^{\prime}}^{r} \frac{c_{D G} e^{-\lambda_{1}\left(t^{\prime}-r\right)}\left|u\left(r+T_{i-1}\left(\theta_{j} \omega\right)\right)-u\left(q+T_{i-1}\left(\theta_{j} \omega\right)\right)\right|}{(r-q)^{\alpha+1}} d q\right)\left(t^{\prime}-r\right)^{\beta^{\prime}+\alpha-1} d r \\
& \leq c \mid\left\|\theta_{T_{i+j-1}} \omega\right\|_{\beta^{\prime}, 0, T\left(\theta_{T_{i+j-1}} \omega\right)}\left(1+\|u\|_{\beta, T_{i-1}\left(\theta_{T_{j}} \omega\right), T_{i}\left(\theta_{T_{j}} \omega\right)}\right)\left(t^{\prime}-s^{\prime}\right)^{\beta^{\prime}} \\
& \leq c \mu\left(1+\|u\|_{\beta, T_{i-1}\left(\theta_{T_{j}} \omega\right), T_{i}\left(\theta_{T_{j}} \omega\right)}\right)(t-s)^{\beta} .
\end{aligned}
$$

Copyright (c) by SIAM. Unauthorized reproduction of this article is prohibited. 
We have used the fact that, by definition, in the interval $\left[0, T_{1}\left(\theta_{T_{i}+j-1} \omega\right)\right]$ the norm of the noise paths is smaller than $\mu$ and that $T_{1}\left(\theta_{T_{i+j-1}} \omega\right) \leq 1$.

Furthermore, for the last term on the right-hand side of (5.2), applying Lemma 3.3 yields that

$$
\begin{aligned}
& \left(t^{\prime}-s^{\prime}\right)^{\beta}\left|\int_{0}^{s^{\prime}} S\left(s^{\prime}-r\right) G\left(u\left(r+T_{i-1}\left(\theta_{T_{j}} \omega\right)\right)\right) d \theta_{T_{i+j-1}} \omega\right|_{V_{\beta}} \\
& \quad \leq\left(t^{\prime}-s^{\prime}\right)^{\beta} c \mu\left(1+\|u\|_{\beta, T_{i-1}\left(\theta_{T_{j}} \omega\right), T_{i}\left(\theta_{T_{j}} \omega\right)}\right) \\
& \quad=(t-s)^{\beta} c \mu\left(1+\|u\|_{\beta, T_{i-1}\left(\theta_{T_{j}} \omega\right), T_{i}\left(\theta_{T_{j}} \omega\right)}\right) .
\end{aligned}
$$

Moreover, for the terms of the sum on (5.1), for $t \in\left[T_{i-1}\left(\theta_{T_{j}} \omega\right), T_{i}\left(\theta_{T_{j}} \omega\right)\right]$,

$$
\begin{aligned}
& \int_{T_{m-1}\left(\theta_{T_{j}} \omega\right)}^{T_{m}\left(\theta_{T_{j}} \omega\right)} S(t-r) G(u(r)) d \theta_{T_{j}} \omega \\
& =\int_{0}^{T_{1}\left(\theta_{T_{m+j-1}} \omega\right)} S\left(t-\left(r+T_{m-1}\left(\theta_{T_{j}} \omega\right)\right)\right) G\left(u\left(r+T_{m-1}\left(\theta_{T_{j}} \omega\right)\right)\right) d \theta_{T_{m+j-1}} \omega \\
& =\int_{0}^{T_{1}\left(\theta_{T_{m+j-1}} \omega\right)} S\left(t^{\prime}+T_{i-1}\left(\theta_{T_{j}} \omega\right)-r-T_{m-1}\left(\theta_{T_{j}} \omega\right)\right) \\
& \quad \times G\left(u\left(r+T_{m-1}\left(\theta_{T_{j}} \omega\right)\right)\right) d \theta_{T_{m+j-1}} \omega \\
& =S\left(t^{\prime}+T_{i-1}\left(\theta_{T_{j}} \omega\right)-T_{m}\left(\theta_{T_{j}} \omega\right)\right) \\
& \quad \times \int_{0}^{T_{1}\left(\theta_{T_{m+j-1}}\right)} S\left(T\left(\theta_{T_{m+j-1}} \omega\right)-r\right) G\left(u\left(r+T_{m-1}\left(\theta_{T_{j}} \omega\right)\right)\right) d \theta_{T_{m+j-1}} \omega
\end{aligned}
$$

for $t^{\prime}=t-T_{i-1}\left(\theta_{T_{j}} \omega\right) \in\left[0, T_{1}\left(\theta_{T_{i+j-1}} \omega\right)\right]$. We have used above that $T_{m-1}\left(\theta_{T_{j}} \omega\right)=$ $T_{m}\left(\theta_{T_{j}} \omega\right)-T_{1}\left(\theta_{T_{m+j-1}} \omega\right)$. Therefore, for $s^{\prime}<t^{\prime} \in\left[0, T_{1}\left(\theta_{T_{j+i-1}} \omega\right)\right]$ we have

$$
\begin{aligned}
& \mid S\left(t^{\prime}+T_{i-1}\left(\theta_{T_{j}} \omega\right)-T_{m}\left(\theta_{T_{j}} \omega\right)\right) \\
& \times \int_{0}^{T_{1}\left(\theta_{T_{m+j-1}}\right)} S\left(T\left(\theta_{T_{m+j-1}} \omega\right)-r\right) G\left(u\left(r+T_{m-1}\left(\theta_{T_{j}} \omega\right)\right)\right) d \theta_{T_{m+j-1}} \omega \\
& -S\left(s^{\prime}+T_{i-1}\left(\theta_{T_{j}} \omega\right)-T_{m}\left(\theta_{T_{j}} \omega\right)\right) \\
& \times \int_{0}^{T_{1}\left(\theta_{T_{m+j-1}}\right)} S\left(T\left(\theta_{T_{m+j-1}} \omega\right)-r\right) G\left(u\left(r+T_{m-1}\left(\theta_{T_{j}} \omega\right)\right)\right) d \theta_{T_{m+j-1}} \omega \mid \\
& \leq \mid\left(S\left(t^{\prime}-s^{\prime}\right)-\mathrm{Id}\right) S\left(s^{\prime}+T_{i-1}\left(\theta_{T_{j}} \omega\right)-T_{m}\left(\theta_{T_{j}} \omega\right)\right) \\
& \times \int_{0}^{T_{1}\left(\theta_{T_{m+j-1}}\right)} S\left(T\left(\theta_{T_{m+j-1}} \omega\right)-r\right) G\left(u\left(r+T_{m-1}\left(\theta_{T_{j}} \omega\right)\right)\right) d \theta_{T_{m+j-1}} \omega \mid \\
& \leq\left(t^{\prime}-s^{\prime}\right)^{\beta} e^{-\lambda_{1}\left(T_{i-1}\left(\theta_{T_{j}} \omega\right)-T_{m}\left(\theta_{T_{j}} \omega\right)\right)} \\
& \times\left|\int_{0}^{T_{1}\left(\theta_{T_{m+j-1}}\right)} S\left(T\left(\theta_{T_{m+j-1}} \omega\right)-r\right) G\left(u\left(r+T_{m-1}\left(\theta_{T_{j}} \omega\right)\right)\right) d \theta_{T_{m+j-1}} \omega\right|_{V_{\beta}} \\
& \leq(t-s)^{\beta} e^{-\lambda_{1}\left(T_{i-1}\left(\theta_{T_{j}} \omega\right)-T_{m}\left(\theta_{T_{j}} \omega\right)\right)} c \mu\left(1+\|u\|_{\beta, T_{m-1}\left(\theta_{T_{j}} \omega\right), T_{m}\left(\theta_{T_{j}} \omega\right)}\right) .
\end{aligned}
$$

Note that to estimate

$$
\sup _{T_{i-1}\left(\theta_{T_{j}} \omega\right) \leq t \leq T_{i}\left(\theta_{T_{j}} \omega\right)}|u(t)|
$$

Copyright $@$ by SIAM. Unauthorized reproduction of this article is prohibited. 


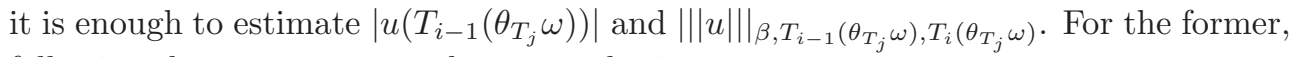
following the same steps as above, we obtain

$$
\begin{aligned}
& \left|u\left(T_{i-1}\left(\theta_{T_{j}} \omega\right)\right)\right| \leq\left|S\left(T_{i-1}\left(\theta_{T_{j}} \omega\right)\right) u_{0}\right|+\left|\int_{0}^{T_{i-1}\left(\theta_{T_{j}} \omega\right)} S\left(T_{i-1}\left(\theta_{T_{j}} \omega\right)-r\right) G(u(r)) d \theta_{T_{j}} \omega\right| \\
& \leq c e^{-\lambda_{1} T_{i-1}\left(\theta_{T_{j}} \omega\right)}\left|u_{0}\right|+\sum_{m=1}^{i-1}\left|\int_{T_{m-1}\left(\theta_{T_{j}} \omega\right)}^{T_{m}\left(\theta_{T_{j}} \omega\right)} S\left(T_{i-1}\left(\theta_{T_{j}} \omega\right)-r\right) G(u(r)) d \theta_{T_{j}} \omega\right| \\
& \leq c e^{-\lambda_{1} T_{i-1}\left(\theta_{T_{j}} \omega\right)}\left|u_{0}\right|+\sum_{m=1}^{i-1} \mid S\left(T_{i-1}\left(\theta_{T_{j}} \omega\right)-T_{m}\left(\theta_{T_{j}} \omega\right)\right) \\
& \quad \times \int_{0}^{T_{1}\left(\theta_{T_{m+j-1}}\right)} S\left(T\left(\theta_{T_{m+j-1}} \omega\right)-r\right) G\left(u\left(r+T_{m-1}\left(\theta_{T_{j}} \omega\right)\right)\right) d \theta_{T_{m+j-1}} \omega \mid \\
& \leq c e^{-\lambda_{1} T_{i-1}\left(\theta_{T_{j}} \omega\right)}\left|u_{0}\right|+c \mu \sum_{m=1}^{i-1} e^{-\lambda_{1}\left(T_{i-1}\left(\theta_{T_{j}} \omega\right)-T_{m}\left(\theta_{T_{j}} \omega\right)\right)} \\
& \quad \times\left(1+\|u\|_{\beta, T_{m-1}\left(\theta_{T_{j}} \omega\right), T_{m}\left(\theta_{T_{j}} \omega\right)}\right) .
\end{aligned}
$$

Collecting all the previous estimates we obtain the conclusion, renaming all appearing constants again as $c$.

\section{REFERENCES}

[1] L. Arnold, Random Dynamical Systems, Springer Monogr. Math., Springer-Verlag, Berlin, 1998.

[2] A. V. Babin And M. I. Vishik, Attractors of Evolution Equations, Stud. Math. Appl. 25, North-Holland, Amsterdam, 1992.

[3] T. Caraballo, M. J. Garrido-Atienza, B. Schmalfuss, and J. Valero, Asymptotic behavior of a stochastic semilinear dissipative functional equation without uniqueness of solutions, Discrete Contin. Dynam. Syst. Ser. B, 14 (2010), pp. 439-455.

[4] C. Castaing and M. Valadier, Convex Analysis and Measurable Multifunctions, Lectures in Math., 580, Springer-Verlag, Berlin, 1977.

[5] Y. Chen, H. Gao, M. J. Garrido-Atienza, and B. Schmalfuss, Pathwise Solutions of SPDEs and Random Dynamical Systems, Discrete Contin. Dyn. Syst., 34 (2014), pp. 79-98.

[6] I. Chueshov, Monotone Random Systems Theory and Applications, Lecture Notes in Math. 1779, Springer-Verlag, Berlin, 2002.

[7] F. Flandoli and B. Schmalfuss, Random attractors for the 3D stochastic Navier-Stokes equation with multiplicative white noise, Stoch. Stoch. Rep., 59 (1996), pp. 21-45.

[8] M. J. Garrido-Atienza, K. Lu, and B. Schmalfuss, Random dynamical systems for stochastic partial differential equations driven by a fractional Brownian motion, Discrete Contin. Dynam. Syst. Ser. B, 14 (2010), pp. 473-493.

[9] M. J. Garrido-Atienza, K. Lu, And B. Schmalfuss, Random dynamical systems for stochastic evolution equations driven by a fractional Brownian motion with Hurst parameter in $(1 / 3,1 / 2]$, in preparation.

[10] M. J. Garrido-Atienza, K. Lu, And B. Schmalfuss, Pathwise Solutions of Stochastic Partial Differential Equations Driven by a Fractional Brownian Motion with Hurst Parameter in (1/3,1/2], arXiv:1205.6735, 2012.

[11] M. J. Garrido-Atienza, K. Lu, And B. Schmalfuss, Compensated fractional derivatives and stochastic evolution equations, C.R. Math., 350 (2012), pp. 1037-1042.

[12] M. J. Garrido-Atienza, B. Maslowski, and B. Schmalfuss, Random attractors for stochastic equations driven by a fractional Brownian motion, Internat. J. Bifur. Chaos, 20 (2010), pp. $1-22$.

[13] M. J. Garrido-Atienza, P. Kloeden, and A. Neuenkirch, Discretization of stationary solutions of stochastic systems driven by fractional Brownian motion, Appl. Math. Optim., 60 (2009), pp. 151-172.

[14] M. J. Garrido-Atienza and B. Schmalfuss, Ergodicity of the infinite dimensional fractional Brownian motion, J. Dynam. Differential Equations, 23 (2011), pp. 671-681.

Copyright $@$ by SIAM. Unauthorized reproduction of this article is prohibited. 
[15] B. GESS, Random attractors for stochastic porous media equations perturbed by space-time linear multiplicative noise, C.R. Acad. Sci. Paris, Ser. I, 350, (2012), pp. 299-302.

[16] B. Gess, W. LiU, And M. Röckner, Random attractors for a class of stochastic partial differential equations driven by general additive noise, J. Differential Equations, 251 (2011), pp. 1225-1253.

[17] A. Gu, Random attractors of stochastic lattice dynamical systems driven by fractional Brownian motion, Internat. J. Bifur. Chaos, 23 (2013), 1350041.

[18] J. K. Hale, Asymptotic Behavior of Dissipative Systems, Math. Surveys Monogr., 25, AMS, Providence, RI, 1988.

[19] H. KunitA, Stochastic Flows and Stochastic Differential Equations, Cambridge University Press, Cambridge, UK, 1990.

[20] B. Maslowski AND D. NuAlart, Evolution equations driven by a fractional Brownian motion, J. Funct. Anal., 202 (2003), pp. 277-305.

[21] B. Maslowski AND B. Schmalfuss, Random dynamical systems and stationary solutions of differential equations driven by the fractional Brownian motion, Stochastic Anal. Appl., 22 (2004), pp. 1577-1607.

[22] S. G. Samko, A. A. Kilbas, And O. I. Marichev, Fractional Integrals and Derivatives: Theory and Applications, Gordon and Breach, Philadelphia, 1993.

[23] B. Schmalfuss, Backward cocycles and attractors of stochastic differential equations, in Proceedings of the International Seminar on Applied Mathematics-Nonlinear Dynamics: Attractor Approximation and Global Behaviour, V. Reitmann, T. Riedrich, and N. Koksch, eds., 1992, pp. 185-192.

[24] B. Schmalfuss, Attractors for the non-autonomous dynamical systems, in Proceedings of EQUADIFF99, K. Gröger, B. Fiedler, and J. Sprekels, eds., World Scientific, Singapore, 2000, pp. 684-690.

[25] R. Temam, Infinite-Dimensional Dynamical Systems in Mechanics and Physics, 2nd ed., Appl. Math. Sci. 68, Springer-Verlag, New York, 1997.

[26] L. C. Young, An integration of Höder type, connected with Stieltjes integration, Acta Math., 67 (1936), pp. 251-282.

[27] M. Z̈̈HLE, Integration with respect to fractal functions and stochastic calculus. I, Probab. Theory Related Fields, 111 (1998), pp. 333-374.

Copyright $@$ by SIAM. Unauthorized reproduction of this article is prohibited. 